\title{
CONTROL FOR THE SINE-GORDON EQUATION
}

\author{
Madalina Petcu ${ }^{1,2}$ and Roger Temam ${ }^{1,3}$
}

\begin{abstract}
In this article we apply the optimal and the robust control theory to the sine-Gordon equation. In our case the control is given by the boundary conditions and we work in a finite time horizon. We present at the beginning the optimal control problem and we derive a necessary condition of optimality and we continue by formulating a robust control problem for which existence and uniqueness of solutions are derived.
\end{abstract}

Mathematics Subject Classification. 35Q53, 49J20, 49J50, 49K20.

Received May 10, 2003. Revised October 2, 2003.

\section{INTRODUCTION}

We consider the damped sine-Gordon equation with non-homogenous Dirichlet boundary conditions, namely

$$
\begin{gathered}
u_{t t}+\alpha u_{t}-u_{x x}+\beta \sin u=0, \quad \text { in } \Omega \times \mathbf{R}_{+}, \quad \Omega=(0, L), \\
u(0, t)=g_{0}(t), \quad u(L, t)=g_{1}(t), \\
u(x, 0)=u_{0}(x), \quad \frac{\partial u}{\partial t}(x, 0)=u_{1}(x) .
\end{gathered}
$$

In physics the sine-Gordon equation is used to model for instance the dynamics of the Josephson junction driven by a current source. This equation has been studied from the point of view of stability of the equation (boundness of trajectories), the existence of absorbing sets and the existence of a global attractor, see e.g. $[12,15]$.

In this article we would like to study the optimal and robust control problems for this equation, when the control is given by the boundary conditions, namely $g_{0}, g_{1}$, in (1.1), see [1,6-8] and [3] for related problems in fluid mechanics.

We are interested in some issues regarding the control of (1.1) when the control is $g=\left(g_{0}, g_{1}\right)$. We will first consider the optimal control problem formulated as follows:

Find a control g minimizing the cost function

$$
\mathcal{J}(g)=\frac{1}{2} \int_{0}^{T}\left|\frac{\partial u_{g}}{\partial t}\right|_{L^{2}(\Omega)}^{2} \mathrm{~d} t+\frac{1}{2} \int_{0}^{T}\left|\frac{\partial u_{g}}{\partial x}\right|_{L^{2}(\Omega)}^{2} \mathrm{~d} t+\frac{l}{2}|g|_{\mathbb{H}^{3}(0, T)}^{2},
$$

\footnotetext{
Keywords and phrases. Robust control, sine-Gordon equation, energy estimates, saddle point.

${ }^{1}$ Laboratoire d'Analyse Numérique, Université de Paris-Sud, Orsay, France; e-mail: madalina.petcu@math.u-psud.fr

2 The Institute of Mathematics of the Romanian Academy, Bucharest, Romania.

3 The Institute for Scientific Computing and Applied Mathematics, Indiana University, Bloomington, IN, USA.
} 
where $g=\left(g_{0}, g_{1}\right)$ and $u_{g}$ is the solution of $(1.1)$ associated with $g$; by $\mathbb{H}^{3}(0, T)$ we denoted $\left(H^{3}(0, T)\right)^{2}$. To guarantee the solvability of $(1.1)$ we require $g(0)=g^{\prime}(0)=0$ and we set

$$
\mathbb{H}_{\Gamma}^{3}(0, T)=\left\{g \in \mathbb{H}^{3}(0, T), g(0)=g^{\prime}(0)=0\right\} .
$$

We obtain the existence of an optimal control in a suitable class and we determine a necessary condition for optimality. This optimal control may not be unique because the optimization problem is nonconvex.

To ensure the uniqueness of the optimal control we find an $l_{0}$ depending on the set on which $g$ is defined and on the initial data such that, for any $l \geq l_{0}$ the cost function will be strictly convex, thus leading to uniqueness.

We also consider a robust control problem for this equation. In this case we write the equation in the form

$$
\begin{aligned}
& \frac{\partial^{2} u}{\partial t^{2}}+\alpha \frac{\partial u}{\partial t}-\frac{\partial^{2} u}{\partial x^{2}}+\beta \sin u=0, \quad \text { in } \quad \Omega \times \mathbf{R}_{+}, \\
& u(0, t)=g_{0}(t)+h_{0}(t), \quad u(L, t)=g_{1}(t)+h_{1}(t), \\
& u(x, 0)=u_{0}(x), \quad \frac{\partial u}{\partial t}(x, 0)=u_{1}(x), \quad x \in \Omega,
\end{aligned}
$$

where the boundary values have been decomposed into the disturbance $h=\left(h_{0}, h_{1}\right)$ and the control $g=\left(g_{0}, g_{1}\right)$; the solution $u$ of $(1.4)$ is also denoted $u(g, h)$ to emphasize its dependence on $g$ and $h$. Mathematically we arrive at a non-differential game for the robust control setting in which a saddle point is sought. Our approach is based on classical existence and characterization results of saddle points in infinite dimensions as given e.g. in [5]. The considered cost function (Lagrangian) reads

$$
\mathcal{J}(g, h)=\frac{1}{2} \int_{0}^{T}\left|\frac{\partial u(g, h)}{\partial t}\right|_{L^{2}(\Omega)}^{2} \mathrm{~d} t+\frac{1}{2} \int_{0}^{T}\left|\frac{\partial u(g, h)}{\partial x}\right|_{L^{2}(\Omega)}^{2} \mathrm{~d} t+\frac{l}{2}|g|_{\mathbb{H}^{3}(0, T)}^{2}-\frac{m}{2}|h|_{\mathbb{H}^{3}(0, T)}^{2},
$$

where $l$ measures the relative price of the control and $m$ measures the relative price of the disturbance. As we explain later on, the aim is now to find the best control $g$ corresponding to the worse disturbance $h$, that is we consider the problem

$$
\inf _{g} \sup _{h} \mathcal{J}(g, h)
$$

$g$ and $h$ belonging to suitable feasible sets.

The content of the article is as follows: in Section 1 we give a short overview of some useful classical results concerning the existence and uniqueness of solution of the sine-Gordon equation. In Section 2.1 we prove the existence, without uniqueness, of a solution for the optimal control problem. In Section 2.2 we derive a necessary condition for optimality using the adjoint state equation; in Section 2.3 we show that by taking $l$ large enough in the cost function (1.2) we obtain the uniqueness of solution of the optimal control problem. Finally, in Section 3, we will see that the robust control problem has a unique solution when $l$ and $m$ appearing in (1.5) are sufficiently large. In the last section we obtain the characterization of the solution of the robust control problem.

We conclude this introduction by recalling well-known results concerning the sine-Gordon equation. We first consider the sine-Gordon equation in the open bounded interval $\Omega=(0, L)$ with homogeneous Dirichlet boundary conditions

$$
\begin{aligned}
& \frac{\partial^{2} u}{\partial t^{2}}+\alpha \frac{\partial u}{\partial t}-\frac{\partial^{2} u}{\partial x^{2}}+\beta \sin u=f, \text { in } \Omega \times \mathbf{R}_{+}, \\
& u(0, t)=0, \quad u(L, t)=0, \\
& u(x, 0)=u_{0}(x), \quad \frac{\partial u}{\partial t}(x, 0)=u_{1}(x), x \in \Omega,
\end{aligned}
$$

where $f$ and $\alpha$ are given, $\alpha>0$. 
We set $H=L^{2}(\Omega), V=H_{0}^{1}(\Omega)$ and we endow these spaces with the usual scalar products and norms.

We write $D(A)=H_{0}^{1}(\Omega) \cap H^{2}(\Omega)$ and, for $u \in D(A)$, we set $A u=-\partial^{2} u / \partial x^{2}$. Then the problem (1.1) is equivalent to the following one:

$$
\begin{aligned}
& u^{\prime \prime}+\alpha u^{\prime}+A u+\beta \sin u=f, \\
& u(0)=u_{0}, \quad u^{\prime}(0)=u_{1},
\end{aligned}
$$

where $\psi^{\prime}:=\partial \psi / \partial t$.

The existence and uniqueness of solution of (1.8) is given by the following result (see e.g. [15]):

Theorem 1.1. Let $\alpha \in \mathbf{R}$ and let $f, u_{0}$ and $u_{1}$ be given satisfying

Then there exists a unique solution $u$ of (1.8) such that

$$
f \in L^{2}([0, T] ; H), \quad u_{0} \in V, \quad u_{1} \in H .
$$

$$
u \in L^{2}([0, T] ; V), \quad u^{\prime} \in L^{2}([0, T] ; H) .
$$

If furthermore, $f^{\prime} \in L^{2}([0, T] ; H), u_{0} \in D(A)$ and $u_{1} \in V$, then $u$ satisfies

$$
u \in L^{2}([0, T] ; D(A)), \quad u^{\prime} \in L^{2}([0, T] ; V) .
$$

For the nonhomogeneous problem (1.1), we have:

Theorem 1.2. Assume that $g \in \mathbb{H}_{\Gamma}^{3}(0, T), u_{0} \in D(A)$ and $u_{1} \in V$. Then there exists a unique solution $u$ of (1.1) with

$$
u \in L^{2}\left([0, T] ; H^{2}(0, L)\right), \quad u^{\prime} \in L^{2}\left([0, T] ; H^{1}(0, L)\right) .
$$

Proof. We construct a lifting function for the boundary conditions, $\phi(x, t)=g_{0}(t)+\left(g_{1}(t)-g_{0}(t)\right)(x / L)$, and we set $v(x, t)=u(x, t)-\phi(x, t)$. Then the system (1.1) is equivalent to the following one:

$$
\begin{aligned}
& \frac{\partial^{2} v}{\partial t^{2}}+\alpha \frac{\partial v}{\partial t}-\frac{\partial^{2} v}{\partial x^{2}}+\beta \sin (v+\phi)=F(x, t), \\
& v(0, t)=0, \quad v(L, t)=0 \\
& v(x, 0)=u_{0}(x), \quad \frac{\partial v}{\partial t}(x, 0)=u_{1}(x)
\end{aligned}
$$

where

$$
F(x, t)=-\left[\frac{\partial^{2} \phi}{\partial t^{2}}+\alpha \frac{\partial \phi}{\partial t}-\frac{\partial^{2} \phi}{\partial x^{2}}\right]=-\left[\frac{\partial^{2} \phi}{\partial t^{2}}+\alpha \frac{\partial \phi}{\partial t}\right]
$$

We derive the a priori estimates on the solutions and using these a priori estimates and the Galerkin method, the proof of the theorem follows.

We multiply $(1.9)_{1}$ by $\partial v / \partial t$ and integrate over $\Omega$. We obtain:

$$
\frac{1}{2} \frac{\mathrm{d}}{\mathrm{d} t}\left|\frac{\partial v}{\partial t}\right|_{L^{2}(\Omega)}^{2}+\alpha\left|\frac{\partial v}{\partial t}\right|_{L^{2}(\Omega)}^{2}+\frac{1}{2} \frac{\mathrm{d}}{\mathrm{d} t}\left|\frac{\partial v}{\partial x}\right|_{L^{2}(\Omega)}^{2}=\int_{\Omega} F(x, t) \frac{\partial v}{\partial t} \mathrm{~d} x-\beta \int_{\Omega} \sin (v+\phi) \frac{\partial v}{\partial t} \mathrm{~d} x .
$$

Using Hölder's inequality and Young's inequality we find:

$$
\left|\int_{\Omega} F(x, t) \frac{\partial v}{\partial t} \mathrm{~d} x\right| \leq c|F(\cdot, t)|_{L^{2}(\Omega)}^{2}+\frac{\alpha}{4}\left|\frac{\partial v}{\partial t}\right|_{L^{2}(\Omega)}^{2},
$$




$$
\left|\int_{\Omega} \beta \sin (v+\phi) \frac{\partial v}{\partial t} \mathrm{~d} x\right| \leq \frac{\alpha}{4}\left|\frac{\partial v}{\partial t}\right|_{L^{2}(\Omega)}^{2}+c \beta^{2}
$$

here and in the sequel $c$ denotes a constant which may be different at different places.

This yields:

$$
\frac{\mathrm{d}}{\mathrm{d} t}\left[\left|\frac{\partial v}{\partial t}\right|_{L^{2}(\Omega)}^{2}+\left|\frac{\partial v}{\partial x}\right|_{L^{2}(\Omega)}^{2}\right]+\alpha\left|\frac{\partial v}{\partial t}\right|_{L^{2}(\Omega)}^{2} \leq c+c|F(\cdot, t)|_{L^{2}(\Omega)}^{2} .
$$

By Gronwall's inequality and some simple computations, we finally obtain:

$$
\left[\left|\frac{\partial v}{\partial t}(t)\right|_{L^{2}(\Omega)}^{2}+\left|\frac{\partial v}{\partial x}(t)\right|_{L^{2}(\Omega)}^{2}\right] \leq c(T), \quad \forall 0<t \leq T
$$

where $c(T)$ is a constant depending on $T$. Further estimates are obtained as follows: we substract $\beta \sin \phi$ from both sides of the first equation (1.9) and write $F_{1}(x, t)=F(x, t)-\beta \sin \phi$.

We call $w=v^{\prime}+\varepsilon v$, where $\varepsilon>0$ will be chosen later on and we take the scalar product of the first equation (1.9) with $A w$. After some easy computations we obtain:

$$
\frac{1}{2} \frac{\mathrm{d}}{\mathrm{d} t}\left[\|w\|^{2}+|A v|^{2}\right]+\varepsilon|A v|^{2}+(\alpha-\varepsilon)\|w\|^{2}-\varepsilon(\alpha-\varepsilon)(v, A w)+\beta((\sin (v+\phi)-\sin \phi, w))=\left(F_{1}, A w\right) .
$$

We know that $|v| \leq c_{1}\|v\|$ for all $v \in V$; using this relation we can write:

$$
\varepsilon|A v|^{2}+(\alpha-\varepsilon)\|w\|^{2}-\varepsilon(\alpha-\varepsilon)(v, A w) \geq \varepsilon|A v|^{2}+(\alpha-\varepsilon)\|w\|^{2}-\varepsilon(\alpha-\varepsilon) c_{1}|A v|\|w\|
$$

Thus we can choose $0<\varepsilon \leq \alpha / 2$ sufficiently small such that

$$
\varepsilon|A v|^{2}+(\alpha-\varepsilon)\|w\|^{2}-\varepsilon(\alpha-\varepsilon)(v, A w) \geq \frac{\varepsilon}{2}|A v|^{2}+\frac{\alpha}{2}\|w\|^{2} .
$$

Applying Young's inequality we see also that:

$$
\begin{aligned}
|\beta((\sin (v+\phi)-\sin \phi, w))| & \leq|\beta|\|\sin (v+\phi)-\sin \phi\|\|w\| \\
& \leq \frac{\alpha}{4}\|w\|^{2}+c\left[\left|\frac{\partial v}{\partial x}+\frac{\partial \phi}{\partial x}\right|^{2}+\left|\frac{\partial \phi}{\partial x}\right|^{2}\right]
\end{aligned}
$$

Writing $\left(F_{1}, A w\right)=\frac{\mathrm{d}}{\mathrm{d} t}\left(F_{1}, A v\right)+\left(\varepsilon F_{1}-F_{1}^{\prime}, A v\right)$, returning to (1.12), and using again Young's inequality we obtain:

$$
\frac{\mathrm{d}}{\mathrm{d} t}\left[\|w\|^{2}+\left|A v-F_{1}\right|^{2}\right]+\frac{\varepsilon}{2}|A v|^{2}+\frac{\alpha}{2}\|w\|^{2} \leq\left|F_{1}^{\prime} \|\right| F_{1}|+| F_{1}-1 /\left.\varepsilon F_{1}^{\prime}\right|^{2}+c\left[\left|\frac{\partial v}{\partial x}+\frac{\partial \phi}{\partial x}\right|^{2}+\left|\frac{\partial \phi}{\partial x}\right|^{2}\right] .
$$

Integrating (1.16) over $(0, t)$, with $0 \leq t \leq T$, and taking into account the previous estimates, we obtain:

$$
\begin{aligned}
\|w(t)\|^{2}+\left|\left(A v-F_{1}\right)(t)\right|^{2} & \leq\|w(0)\|^{2}+\left|\left(A v-F_{1}\right)(0)\right|^{2}+c \\
& =\left\|u_{1}+\varepsilon u_{0}\right\|^{2}+\left|A u_{0}-F(0)\right|^{2}+c
\end{aligned}
$$

for all $t>0$. In (1.17) $c$ depends on the data but not on $T$. We obtained a priori estimates for $u$ in $L^{2}\left(0, T ; H^{2}(0, L)\right)$ and $u^{\prime}$ in $L^{2}\left(0, T ; H^{1}(0, L)\right)$. 


\section{The optimal CONTRol PRoblem}

We consider equation (1.1) as the state equation where $g(t)=\left(g_{0}(t), g_{1}(t)\right)$ is the control function. We formulate the control problem as follows:

$$
\mathcal{P} \mid \begin{gathered}
\text { Find a function } g \in \mathbb{H}_{\Gamma}^{3}(0, T) \text { minimizing the cost function defined as } \\
\mathcal{J}(g)=\frac{1}{2} \int_{0}^{T}\left|\frac{\partial u_{g}}{\partial t}\right|_{L^{2}(\Omega)}^{2} \mathrm{~d} t+\frac{1}{2} \int_{0}^{T}\left|\frac{\partial u_{g}}{\partial x}\right|_{L^{2}(\Omega)}^{2} \mathrm{~d} t+\frac{l}{2}|g|_{\mathbb{H}_{\Gamma}^{3}(0, T)}^{2} .
\end{gathered}
$$

\subsection{Existence of solutions}

Problem $\mathcal{P}$ is a nonconvex optimization problem; existence of an optimal pair $\left(\bar{g}, \bar{u}_{g}\right)$ is stated as follows:

Theorem 2.1. Let there be given $u_{0} \in D(A), u_{1} \in V$. Then there exists at least one pair $\bar{g} \in \mathbb{H}_{\Gamma}^{3}(0, T)$ and $\bar{u}:=u_{\bar{g}} \in L^{2}\left([0, T] ; H^{2}(\Omega)\right)$ with $\bar{u}^{\prime} \in L^{2}\left([0, T] ; H^{1}(\Omega)\right)$, such that the functional $\mathcal{J}(g)$ attains its minimum at $\bar{g}$ and $\bar{u}$ is the solution of system (1.1) corresponding to $\bar{g}$.

Proof. Let $\lambda=\inf _{g \in \mathbb{H}_{\Gamma}^{3}(0, T)} \mathcal{J}(g)$ and let $\left(g_{n}\right)_{n}$ be a minimizing sequence for problem $\mathcal{P}$. We denote by $u_{n}=u_{g_{n}}$ and $v_{n}=v_{g_{n}}$ the corresponding solutions of systems (1) and respectively (1.9).

We observe that $\left|g_{n}\right|_{\mathbb{H}^{3}(0, T)}^{2} \leq \mathcal{J}\left(g_{n}\right)$, which implies that $\left(g_{n}\right)_{n} \subset \mathbb{H}_{\Gamma}^{3}(0, T)$ is a bounded sequence in $\mathbb{H}^{3}(0, T)$. Hence there exist $\bar{g} \in \mathbb{H}_{\Gamma}^{3}(0, T)$ and a subsequence, still denoted $g_{n}$, such that

$$
g_{n} \rightarrow \bar{g} \text { weakly in } \mathbb{H}^{3}(0, T) .
$$

We call $\phi_{n}(x, t)=g_{n, 0}(t)+\left(g_{n, 1}(t)-g_{n, 0}(t)\right)(x / L)$ and $\bar{\phi}(x, t)=\bar{g}_{0}(t)+\left(\bar{g}_{1}(t)-\bar{g}_{0}(t)\right)(x / L)$ the corresponding lifting functions and we know that $v_{n}$ satisfies the following equations:

$$
\begin{aligned}
& \frac{\partial^{2} v_{n}}{\partial t^{2}}+\alpha \frac{\partial v_{n}}{\partial t}-\frac{\partial^{2} v_{n}}{\partial x^{2}}+\beta \sin \left(v_{n}+\phi_{n}\right)=F_{n}(x, t), \\
& v_{n}(0, t)=0, \quad v_{n}(L, t)=0, \\
& v_{n}(x, 0)=u_{0}(x), \quad \frac{\partial v_{n}}{\partial t}(x, 0)=u_{1}(x),
\end{aligned}
$$

where

$$
F_{n}(x, t)=-\left[\frac{\partial^{2} \phi_{n}}{\partial t^{2}}+\alpha \frac{\partial \phi_{n}}{\partial t}-\frac{\partial^{2} \phi_{n}}{\partial x^{2}}\right]=-\left[\frac{\partial^{2} \phi_{n}}{\partial t^{2}}+\alpha \frac{\partial \phi_{n}}{\partial t}\right] .
$$

Using the fact that $g_{n}$ is bounded in $\mathbb{H}^{3}(0, T)$, we derive the same kind of estimates as in the proof of Theorem 1.2 by exactly the same method, namely we multiply $(2.2)_{1}$ by $\partial v_{n} / \partial t$, integrate over $\Omega$ and apply Gronwall's inequality. We obtain:

$$
\begin{gathered}
\left(v_{n}\right)_{n} \text { is bounded in } L^{\infty}(0, T ; V), \\
\left(\frac{\partial v_{n}}{\partial t}\right)_{n} \text { is bounded in } L^{\infty}(0, T ; H) .
\end{gathered}
$$

For stronger estimates we substract $\beta \sin \phi_{n}$ from each side of $(2.2)$, set $F_{1, n}(x, t)=F_{n}(x, t)-\beta \sin \phi_{n}$, we introduce $w_{n}=v_{n}^{\prime}+\varepsilon v_{n}$, where $\varepsilon$ is exactly as in (1.13) and take the scalar product in $H$ of the equation obtained with $A w_{n}$. After computations identical to those of Theorem 1.2 and remembering that $\phi_{n}$ is bounded in $\mathbb{H}^{3}(0, T)$ we see that, as $n \rightarrow \infty$,

$$
\begin{aligned}
& \left(w_{n}\right)_{n} \text { remains bounded in } L^{\infty}(0, T ; V), \\
& \left(A v_{n}-F_{1, n}\right)_{n} \text { remains bounded in } L^{\infty}\left(0, T ; L^{2}(\Omega)\right) ;
\end{aligned}
$$


taking into account the form of $F_{1, n}$ and (2.3) we deduce that, as $n \rightarrow \infty$,

$$
\begin{aligned}
& \left(v_{n}\right)_{n} \text { remains bounded in } L^{\infty}(0, T ; D(A)), \\
& \left(v_{n}^{\prime}\right)_{n} \text { remains is bounded in } L^{\infty}(0, T ; V) .
\end{aligned}
$$

Passing to a subsequence, still denoted $v_{n}$ we see that:

$$
\begin{aligned}
& v_{n} \rightarrow \bar{v} \text { in } L^{\infty}(0, T ; D(A)) \text { weak-star, } \\
& \frac{\partial v_{n}}{\partial t} \rightarrow \frac{\partial \bar{v}}{\partial t} \text { in } L^{\infty}(0, T ; V) \text { weak-star, }
\end{aligned}
$$

where $\bar{v} \in L^{\infty}(0, T ; D(A)), \bar{v}^{\prime} \in L^{\infty}(0, T ; V)$.

We infer from $(2.9),(2.10)$ and a compactness theorem in $[10,11]$ (see also [15]), that $v_{n} \rightarrow \bar{v}$ strongly in $L^{2}(0, T ; H)$. Also, since the sequence $\left(g_{n}\right)_{n}$ is bounded in $\left(H_{0}^{1}(0, T)\right)^{2}$, we can choose the subsequence $n$ so that $g_{n} \rightarrow \bar{g}$ strongly in $\left(L^{2}(0, T)\right)^{2}$.

By the expression of $\phi_{n}$ we see that $\phi_{n} \rightarrow \bar{\phi}$ strongly in $L^{2}(0, T ; H)$ and thus $u_{n} \rightarrow \bar{u}$ strongly in $L^{2}(0, T ; H)$.

We also notice that

$$
\begin{aligned}
& \frac{\partial u_{n}}{\partial t} \rightarrow \frac{\partial \bar{u}}{\partial t} \text { weakly in } L^{2}\left(0, T ; H^{1}(\Omega)\right) \\
& \frac{\partial u_{n}}{\partial x} \rightarrow \frac{\partial \bar{u}}{\partial x} \text { weakly in } L^{2}\left(0, T ; H^{1}(\Omega)\right) .
\end{aligned}
$$

It is easy to see that $\bar{u}$ is a solution of system (1.1) corresponding to $\bar{g}$ or equivalently that $\bar{v}$ is solution of the corresponding system (1.9): indeed since $v_{n} \rightarrow \bar{v}$ and $\phi_{n} \rightarrow \bar{\phi}$ strongly in $L^{2}(0, T ; H)$ we see that:

$$
\sin \left(v_{n}+\phi_{n}\right) \rightarrow \sin (\bar{v}+\bar{\phi}) \text { strongly in } L^{2}(0, T ; H) .
$$

Next we pass to the limit in (2.2); we find that $\bar{v}$ is solution of (1.9) with $F$ replaced by $\bar{F}$ where $\bar{F}=$ $-\left[\partial^{2} \bar{\phi} / \partial t^{2}+\alpha \partial \bar{\phi} / \partial t\right]$. To conclude the proof we use the lower semi-continuity of the norm and we obtain that $\mathcal{J}(\bar{g}) \leq \liminf _{n} \mathcal{J}\left(g_{n}\right)=\lambda$ and thus, $\mathcal{J}(\bar{g})=\lambda$.

Remark 2.2. Although this result is not relevant to our purpose, let us note (see e.g. [13]) that stronger convergence results than those inferred from (2.9) and (2.10) hold; in particular $v_{n}$ converges to $\bar{v}$ strongly in $L^{2}(0, T ; V)$ and $\partial v_{n} / \partial t$ converges to $\partial v / \partial t$ strongly in $L^{2}(0, T ; H)$ (and more).

\subsection{The adjoint state}

In this section we observe that the cost function $\mathcal{J}$ is Gâteaux differentiable and using the fact that $\mathcal{J}^{\prime}(\bar{g})=0$ we derive the Necessary Condition for Optimality (NCO) for the control problem $\mathcal{P}$. We set

$$
\mathcal{H}:=\left\{u \in L^{2}\left(0, T ; H^{2}(\Omega)\right), u^{\prime} \in L^{2}\left(0, T ; H^{1}(\Omega)\right)\right\}
$$

$\mathcal{H}$ is endowed with the norm

$$
|u|_{\mathcal{H}}:=\left\{|u|_{L^{2}\left(0, T ; H^{2}(\Omega)\right)}^{2}+\left|u^{\prime}\right|_{L^{2}\left(0, T ; H^{1}(\Omega)\right)}^{2}\right\}^{1 / 2} .
$$

Lemma 2.1. Let $u_{0} \in D(A)$ and $u_{1} \in V$. Then the mapping $g \mapsto u_{g}$ from $\mathbb{H}_{\Gamma}^{3}(0, T)$ into $\mathcal{H}$ is Gâteaux differentiable. Furthermore its directional derivative $\left(D u_{g} / D g\right)(\varphi):=w(\varphi)$ at $g$ in direction $\varphi=\left(\varphi_{0}, \varphi_{1}\right)$ is the 
solution of the linearized problem:

$$
\begin{aligned}
& \frac{\partial^{2} w}{\partial t^{2}}+\alpha \frac{\partial w}{\partial t}-\frac{\partial^{2} w}{\partial x^{2}}+\beta w \cos u_{g}=0 \\
& w(0, t)=\varphi_{0}, \quad w(L, t)=\varphi_{1} \\
& w(x, 0)=0, \quad \frac{\partial w}{\partial t}(x, 0)=0 .
\end{aligned}
$$

Proof. We fix $u_{0} \in D(A), u_{1} \in V$ and let $g, \varphi \in \mathcal{F}$. We need to prove the following:

$$
\lim _{\lambda \rightarrow 0} \frac{\left|u_{g+\lambda \varphi}-u_{g}-\lambda w(\varphi)\right|_{\mathcal{H}}}{|\lambda|}=0
$$

We set $R=u_{g+\lambda \varphi}-u_{g}-\lambda w(\varphi) ; R$ is solution of the following problem:

$$
\begin{aligned}
& \frac{\partial^{2} R}{\partial t^{2}}+\alpha \frac{\partial R}{\partial t}-\frac{\partial^{2} R}{\partial x^{2}}+\beta\left[\sin u_{g+\lambda \varphi}-\sin u_{g}-\lambda w \cos u_{g}\right]=0 \\
& R(0, t)=0, \quad R(L, t)=0 \\
& R(x, 0)=0, \quad \frac{\partial R}{\partial t}(x, 0)=0 .
\end{aligned}
$$

We take the scalar product in $L^{2}(\Omega)$ of the first equation $(2.16)$ with $\partial R / \partial t$; we obtain:

$$
\frac{1}{2} \frac{\mathrm{d}}{\mathrm{d} t}\left[\left|\frac{\partial R}{\partial t}\right|_{L^{2}(\Omega)}^{2}+\left|\frac{\partial R}{\partial x}\right|_{L^{2}(\Omega)}^{2}\right]+\alpha\left|\frac{\partial R}{\partial t}\right|_{L^{2}(\Omega)}^{2}=I_{1}+I_{2}
$$

where we denoted:

$$
\begin{aligned}
& I_{1}=-\int_{\Omega}\left[\sin u_{g+\lambda \varphi}-\sin u_{g}-\cos u_{g}\left(u_{g+\lambda \varphi}-u_{g}\right)\right] \frac{\partial R}{\partial t} \mathrm{~d} x \\
& I_{2}=-\beta \int_{\Omega}\left(u_{g+\lambda \varphi}-u_{g}-\lambda w(\varphi)\right) \cos u_{g} \frac{\partial R}{\partial t} \mathrm{~d} x
\end{aligned}
$$

We estimate $I_{2}$ as:

$$
\left|I_{2}\right| \leq \frac{\alpha}{4}\left|\frac{\partial R}{\partial t}\right|_{L^{2}(\Omega)}^{2}+c|R|_{L^{2}(\Omega)}^{2}
$$

To estimate $I_{1}$, we first prove that

$$
\left|u_{g+\lambda \varphi}-u_{g}\right|_{\mathcal{C}(\bar{\Omega})} \leq c|\lambda|
$$

We know that

$$
\begin{aligned}
\left|u_{g+\lambda \varphi}-u_{g}\right|_{\mathcal{C}(\bar{\Omega})} & =\left|v_{g+\lambda \varphi}-v_{g}+\phi_{g+\lambda \varphi}-\phi_{g}\right|_{\mathcal{C}(\bar{\Omega})} \\
& \leq\left|v_{g+\lambda \varphi}-v_{g}\right|_{\mathcal{C}(\bar{\Omega})}+\left|\phi_{\lambda \varphi}\right|_{\mathcal{C}(\bar{\Omega})} \\
& =|\hat{v}|_{\mathcal{C}(\bar{\Omega})}+\lambda\left|\phi_{\varphi}\right|_{\mathcal{C}(\bar{\Omega})}
\end{aligned}
$$

where we denoted $\hat{v}:=v_{g+\lambda \varphi}-v_{g}$. 
The function $\hat{v}$ satisfies the equations:

$$
\begin{aligned}
& \frac{\partial^{2} \hat{v}}{\partial t^{2}}+\alpha \frac{\partial \hat{v}}{\partial t}-\frac{\partial^{2} \hat{v}}{\partial x^{2}}+\beta\left[\sin v_{g+\lambda \varphi}-\sin v_{g}\right]=\lambda F_{\varphi} \\
& \hat{v}(0, t)=0, \quad \hat{v}(L, t)=0 \\
& \hat{v}(x, 0)=\lambda \phi_{\varphi}, \quad \frac{\partial \hat{v}}{\partial t}(x, 0)=\lambda \frac{\partial \phi_{\varphi}}{\partial t}
\end{aligned}
$$

We take the scalar product in $L^{2}(\Omega)$ of the first equation (2.21) with $\partial \hat{v} / \partial t$ and we obtain:

$$
\frac{1}{2} \frac{\mathrm{d}}{\mathrm{d} t}\left[\left|\frac{\partial \hat{v}}{\partial t}\right|_{L^{2}(\Omega)}^{2}+\left|\frac{\partial \hat{v}}{\partial x}\right|_{L^{2}(\Omega)}^{2}\right]+\alpha\left|\frac{\partial \hat{v}}{\partial t}\right|_{L^{2}(\Omega)}^{2}+\beta \int_{\Omega}\left[\sin v_{g+\lambda \varphi}-\sin v_{g}\right] \frac{\partial \hat{v}}{\partial t} \mathrm{~d} x=\lambda \int_{\Omega} F_{\varphi} \frac{\partial \hat{v}}{\partial t} \mathrm{~d} x .
$$

Hence using the Poincaré inequality we find:

$$
\begin{aligned}
\left|\beta \int_{\Omega}\left[\sin v_{g+\lambda \varphi}-\sin v_{g}\right] \frac{\partial \hat{v}}{\partial t} \mathrm{~d} x\right| & \leq|\beta| \int_{\Omega}\left|v_{g+\lambda \varphi}-v_{g}\right|\left|\frac{\partial \hat{v}}{\partial t}\right| \mathrm{d} x \\
& \leq|\beta| \int_{\Omega}|\hat{v}|\left|\frac{\partial \hat{v}}{\partial t}\right| \mathrm{d} x \\
& \leq \frac{\alpha}{4}\left|\frac{\partial \hat{v}}{\partial t}\right|_{L^{2}(\Omega)}^{2}+c\left|\frac{\partial \hat{v}^{2}}{\partial x}\right|_{L^{2}(\Omega)}
\end{aligned}
$$

We also estimate:

$$
\left|\lambda \int_{\Omega} F_{\varphi} \frac{\partial \hat{v}}{\partial t} \mathrm{~d} x\right| \leq \frac{\alpha}{4}\left|\frac{\partial \hat{v}}{\partial t}\right|_{L^{2}(\Omega)}^{2}+\lambda^{2} c\left|F_{\varphi}\right|_{L^{2}(\Omega)}^{2}
$$

Returning to (2.22) we write

$$
\frac{1}{2} \frac{\mathrm{d}}{\mathrm{d} t}\left[\left|\frac{\partial \hat{v}}{\partial t}\right|_{L^{2}(\Omega)}^{2}+\left|\frac{\partial \hat{v}}{\partial x}\right|_{L^{2}(\Omega)}^{2}\right]+\frac{\alpha}{2}\left|\frac{\partial \hat{v}}{\partial t}\right|_{L^{2}(\Omega)}^{2} \leq c\left|\frac{\partial \hat{v}}{\partial x}\right|_{L^{2}(\Omega)}^{2}+\lambda^{2} c\left|F_{\varphi}\right|_{L^{2}(\Omega)}^{2} .
$$

Using the Gronwall's lemma we obtain

$$
\left|\frac{\partial \hat{v}}{\partial t}(t)\right|_{L^{2}(\Omega)}^{2}+\left|\frac{\partial \hat{v}}{\partial x}(t)\right|_{L^{2}(\Omega)}^{2} \leq \lambda^{2} c \int_{0}^{T}\left|F_{\varphi}\right|_{L^{2}(\Omega)}^{2} \mathrm{~d} t
$$

for all $t \leq T$. Here $\int_{0}^{T}\left|F_{\varphi}\right|_{L^{2}(\Omega)}^{2} \mathrm{~d} t$ is a constant independent of $\lambda$, so we have

$$
\left|\frac{\partial \hat{v}}{\partial x}(t)\right|_{L^{2}(\Omega)} \leq c \lambda
$$

Remembering that $\hat{v} \in H_{0}^{1}(\Omega) \subset \mathcal{C}(\bar{\Omega})$, we obtain $|\hat{v}(x, t)| \leq c \lambda$ for all $x \in \bar{\Omega}$, and $0<t<T$. Returning to (2.20) we see that

$$
\left|u_{g+\lambda \varphi}(x, t)-u_{g}(x, t)\right| \leq \lambda c, \quad \forall x \in \bar{\Omega}, \quad 0<t<T .
$$

We know that

$$
\left|\sin u_{g+\lambda \varphi}-\sin u_{g}-\left(u_{g+\lambda \varphi}-u_{g}\right) \cos u_{g}\right| \leq\left|u_{g+\lambda \varphi}-u_{g}\right|^{2}
$$


for all $x \in \Omega$, and $0<t<T$ :

$$
\begin{aligned}
\left|I_{1}\right| & \leq c \int_{\Omega}\left|u_{g+\lambda \varphi}-u_{g}\right|^{2}\left|\frac{\partial R}{\partial t}\right| \mathrm{d} x \\
& \leq c\left|u_{g+\lambda \varphi}-u_{g}\right|_{L^{\infty}(\Omega)}\left|u_{g+\lambda \varphi}-u_{g}\right|_{L^{2}(\Omega)}\left|\frac{\partial R}{\partial t}\right|_{L^{2}(\Omega)} \\
& \leq c \lambda^{4}+\left|\frac{\partial R}{\partial t}\right|_{L^{2}(\Omega)}^{2}
\end{aligned}
$$

With these estimates (2.17) becomes:

$$
\frac{1}{2} \frac{\mathrm{d}}{\mathrm{d} t}\left[\left|\frac{\partial R}{\partial t}\right|_{L^{2}(\Omega)}^{2}+\left|\frac{\partial R}{\partial x}\right|_{L^{2}(\Omega)}^{2}\right]+\alpha\left|\frac{\partial R}{\partial t}\right|_{L^{2}(\Omega)}^{2} \leq c \lambda^{4}+c\left[\left|\frac{\partial R}{\partial t}\right|_{L^{2}(\Omega)}^{2}+\left|\frac{\partial R}{\partial x}\right|_{L^{2}(\Omega)}^{2}\right] .
$$

Hence, using Gronwall's inequality we obtain:

$$
\left|\frac{\partial R}{\partial t}(t)\right|_{L^{2}(\Omega)}^{2}+\left|\frac{\partial R}{\partial x}(t)\right|_{L^{2}(\Omega)}^{2} \leq c \lambda^{4}, \text { for all } t \leq T
$$

For stronger estimates we multiply $(2.16)$ by $\partial^{3} R / \partial x^{2} \partial t$, integrate over $\Omega$ and obtain:

$$
\frac{1}{2} \frac{\mathrm{d}}{\mathrm{d} t}\left[\left|\frac{\partial^{2} R}{\partial x \partial t}\right|_{L^{2}}^{2}+\left|\frac{\partial^{2} R}{\partial x^{2}}\right|_{L^{2}}^{2}\right]+\alpha\left|\frac{\partial^{2} R}{\partial x \partial t}\right|_{L^{2}}^{2}+\beta \int_{\Omega}\left[\sin u_{g+\lambda \varphi}-\sin u_{g}-\lambda w \cos u_{g}\right] \frac{\partial^{3} R}{\partial^{2} x \partial t} \mathrm{~d} x=0 .
$$

We notice that:

$$
\begin{aligned}
\int_{\Omega}\left[\sin u_{g+\lambda \varphi}-\sin u_{g}-\lambda w \cos u_{g}\right] & \frac{\partial^{3} R}{\partial^{2} x \partial t} \mathrm{~d} x=\frac{\mathrm{d}}{\mathrm{d} t}\left(\sin u_{g+\lambda \varphi}-\sin u_{g}-\lambda w \cos u_{g}, \frac{\partial^{2} R}{\partial x^{2}}\right)_{L^{2}} \\
& -\int_{\Omega}\left[u_{g+\lambda \varphi}^{\prime} \cos u_{g+\lambda \varphi}-u_{g}^{\prime} \cos u_{g}-\lambda w^{\prime} \cos u_{g}+\lambda w u_{g}^{\prime} \sin u_{g}\right] \frac{\partial^{2} R}{\partial x^{2}} \mathrm{~d} x
\end{aligned}
$$

Using (2.32) in (2.31) we find:

$$
\begin{aligned}
\frac{\mathrm{d}}{\mathrm{d} t}\left[\frac{1}{2}\left|\frac{\partial^{2} R}{\partial x \partial t}\right|_{L^{2}}^{2}+\frac{1}{2}\left|\frac{\partial^{2} R}{\partial x^{2}}\right|_{L^{2}}^{2}+\right. & \left.\beta\left(\sin u_{g+\lambda \varphi}-\sin u_{g}-\lambda w \cos u_{g}, \frac{\partial^{2} R}{\partial x^{2}}\right)_{L^{2}}\right]+\alpha\left|\frac{\partial^{2} R}{\partial x \partial t}\right|_{L^{2}}^{2} \\
& =\beta \int_{\Omega}\left[\cos u_{g+\lambda \varphi} u_{g+\lambda \varphi}^{\prime}-\cos u_{g} u_{g}^{\prime}-\lambda w^{\prime} \cos u_{g}+\lambda w u_{g}^{\prime} \sin u_{g}\right] \frac{\partial^{2} R}{\partial x^{2}} \mathrm{~d} x
\end{aligned}
$$

We remark that:

$$
\begin{array}{r}
\left|\left(\sin u_{g+\lambda \varphi}-\sin u_{g}-\lambda w \cos u_{g}, \frac{\partial^{2} R}{\partial x^{2}}\right)_{L^{2}}\right| \leq\left|\left(\sin u_{g+\lambda \varphi}-\sin u_{g}-\cos u_{g}\left(u_{g+\lambda \varphi}-u_{g}\right), \frac{\partial^{2} R}{\partial x^{2}}\right)_{L^{2}}\right| \\
+\left|\left(\cos u_{g}\left(u_{g+\lambda \varphi}-u_{g}-\lambda w\right), \frac{\partial^{2} R}{\partial x^{2}}\right)\right|
\end{array}
$$

Using the same arguments as before we find:

$$
\left|\left(\sin u_{g+\lambda \varphi}-\sin u_{g}-\lambda w \cos u_{g}, \frac{\partial^{2} R}{\partial x^{2}}\right)_{L^{2}}\right| \leq c_{0}^{\prime} \lambda^{2}\left|\frac{\partial^{2} R}{\partial x^{2}}\right|_{L^{2}} .
$$


We notice that

$$
\frac{1}{2}\left|\frac{\partial^{2} R}{\partial x \partial t}\right|_{L^{2}}^{2}+\frac{1}{2}\left|\frac{\partial^{2} R}{\partial x^{2}}\right|_{L^{2}}^{2}+\beta\left(\sin u_{g+\lambda \varphi}-\sin u_{g}-\lambda w \cos u_{g}, \frac{\partial^{2} R}{\partial x^{2}}\right)_{L^{2}}+c^{\prime 2}{ }_{1}^{2} \lambda^{4} \geq \frac{1}{2}\left|\frac{\partial^{2} R}{\partial x \partial t}\right|_{L^{2}}^{2}+\frac{1}{4}\left|\frac{\partial^{2} R}{\partial x^{2}}\right|_{L^{2}}^{2},
$$

so we write

$$
\begin{aligned}
\frac{\mathrm{d}}{\mathrm{d} t}\left[\frac{1}{2}\left|\frac{\partial^{2} R}{\partial x \partial t}\right|_{L^{2}}^{2}+\right. & \left.\frac{1}{2}\left|\frac{\partial^{2} R}{\partial x^{2}}\right|_{L^{2}}^{2}+\beta\left(\sin u_{g+\lambda \varphi}-\sin u_{g}-\lambda w \cos u_{g}, \frac{\partial^{2} R}{\partial x^{2}}\right)_{L^{2}}+c_{1}^{\prime 2} \lambda^{4}\right] \\
& +\alpha\left|\frac{\partial^{2} R}{\partial x \partial t}\right|_{L^{2}}^{2}=\beta \int_{\Omega}\left[\cos u_{g+\lambda \varphi} u_{g+\lambda \varphi}^{\prime}-\cos u_{g} u_{g}^{\prime}-\lambda w^{\prime} \cos u_{g}+\lambda w u_{g}^{\prime} \sin u_{g}\right] \frac{\partial^{2} R}{\partial x^{2}} \mathrm{~d} x
\end{aligned}
$$

We now need to estimate the RHS of (2.37). We notice that:

$$
\begin{array}{r}
u_{g+\lambda \varphi}^{\prime} \cos u_{g+\lambda \varphi}-u_{g}^{\prime} \cos u_{g}+\lambda w u_{g}^{\prime} \sin u_{g}=u_{g+\lambda \varphi}^{\prime}\left[\cos u_{g+\lambda \varphi}-\cos u_{g}+\left(u_{g+\lambda \varphi}-u_{g}\right) \sin u_{g}\right] \\
-\left(u_{g+\lambda \varphi}-u_{g}\right)\left(u_{g+\lambda \varphi}^{\prime}-u_{g}^{\prime}\right) \sin u_{g}-u_{g}^{\prime}\left(u_{g+\lambda \varphi}-u_{g}-\lambda w\right) \sin u_{g}+R^{\prime} \cos u_{g}
\end{array}
$$

where $R^{\prime}=\partial R / \partial t$. Using the same kind of estimates as before we find:

$$
|R H S| \leq c \lambda^{4}+\frac{1}{4}\left|\frac{\partial^{2} R}{\partial x^{2}}\right|_{L^{2}}^{2}+c\left|R^{\prime}\right|_{L^{2}}^{2} .
$$

Using Gronwall's lemma we obtain:

$$
\left|\frac{\partial^{2} R}{\partial x \partial t}\right|_{L^{2}}^{2}+\left|\frac{\partial^{2} R}{\partial x^{2}}\right|_{L^{2}}^{2} \leq c \lambda^{4} .
$$

This implies that $|R|_{\mathcal{H}} \leq c \lambda^{2}, \mathcal{H}$ as in (2.13') with the norm given by (2.14), and thus

$$
\lim _{\lambda \rightarrow 0} \frac{|R|_{\mathcal{H}}}{\lambda}=0
$$

We can now state and prove our main result from this section:

Theorem 2.3 (necessary condition of optimality-NCO). Let $(\bar{g}, \bar{u})$ be an optimal pair of problem $(P)$; then the following NCO holds in $\left(\mathbb{H}_{\Gamma}^{3}(0, T)\right)^{\prime}$ that is the dual of $\left(\mathbb{H}_{\Gamma}^{3}(0, T)\right)$ :

$$
\tau \bar{u}+\tau \hat{\bar{w}}+l \Lambda \bar{g}=0
$$

where $\Lambda$ is the canonical isomorphism of $\mathbb{H}_{\Gamma}^{3}(0, T)$ onto $\left(\mathbb{H}_{\Gamma}^{3}(0, T)\right)^{\prime}{ }^{1}$.

In (2.40), $(\bar{u}, \hat{\bar{w}})$ is the solution of the following system

$$
\begin{aligned}
& \frac{\partial^{2} u}{\partial t^{2}}+\alpha \frac{\partial u}{\partial t}-\frac{\partial^{2} u}{\partial x^{2}}+\beta \sin u=0, \\
& \frac{\partial^{2} \hat{w}}{\partial t^{2}}-\alpha \frac{\partial \hat{w}}{\partial t}-\frac{\partial^{2} \hat{w}}{\partial x^{2}}+\beta \hat{w} \cos u=\frac{\partial^{2} u}{\partial t^{2}}+\frac{\partial^{2} u}{\partial x^{2}}, \\
& u(0, t)=g_{0}, \quad u(L, t)=g_{1}, \quad \hat{w}(0, t)=0, \quad \hat{w}(L, t)=0, \\
& u(x, 0)=u_{0}, \quad \frac{\partial u}{\partial t}(x, 0)=u_{1}, \quad \hat{w}(x, T)=0, \quad \frac{\partial \hat{w}}{\partial t}(x, T)=\frac{\partial u}{\partial t}(x, T) ;
\end{aligned}
$$

\footnotetext{
${ }^{1}$ The operator $\Lambda$ can be "explicitly" defined by the solution of a boundary value problem which depends on the norm endowing $\mathbb{H}_{\Gamma}^{3}(0, T)$; which could be the norm of $H^{3}(0, T)$ or

$$
g \rightarrow\left(\left|g^{\prime \prime}(0)\right|^{2}+\left|g^{\prime \prime \prime}\right|_{L^{2}(0, T)}^{2}\right)^{1 / 2} .
$$
}


$\tau$ is the linear operator from $H^{2}(\Omega)$ into $\mathbb{R}^{2}$ defined by:

$$
u \rightarrow \tau u=\left(-\frac{\partial u}{\partial x}(0), \frac{\partial u}{\partial x}(L)\right) .
$$

Proof. Let $(\bar{g}, \bar{u})$ be an optimal pair. We know then that $(D \mathcal{J} / D g)(\bar{g})=0$.

$$
\begin{array}{r}
\frac{D \mathcal{J}}{D g}(g) \cdot \varphi=\int_{0}^{T}\left(\frac{\partial u}{\partial t}, \frac{\partial w}{\partial t}\right)_{L^{2}(\Omega)} \mathrm{d} t+\int_{0}^{T}\left(\frac{\partial u}{\partial x}, \frac{\partial w}{\partial x}\right)_{L^{2}(\Omega)} \mathrm{d} t \\
+l \int_{0}^{T}\left(g \cdot \varphi+\frac{\mathrm{d} g}{\mathrm{~d} t} \cdot \frac{\mathrm{d} \varphi}{\mathrm{d} t}+\frac{\mathrm{d}^{2} g}{\mathrm{~d} t^{2}} \cdot \frac{\mathrm{d}^{2} \varphi}{\mathrm{d} t^{2}}+\frac{\mathrm{d}^{3} g}{\mathrm{~d} t^{3}} \cdot \frac{\mathrm{d}^{3} \varphi}{\mathrm{d} t^{3}}\right) \mathrm{d} t
\end{array}
$$

where $w(\varphi)=(D \mathcal{J} / D g)(\varphi)$ is the solution of $(2.15)$.

Integrating by parts we obtain:

$$
\begin{aligned}
\int_{0}^{T}\left(\frac{\partial u}{\partial x}, \frac{\partial w}{\partial x}\right)_{L^{2}(\Omega)} \mathrm{d} t & =\int_{0}^{T} \int_{0}^{L} \frac{\partial u}{\partial x} \cdot \frac{\partial w}{\partial x} \mathrm{~d} x \mathrm{~d} t=\int_{0}^{T} \frac{\partial u}{\partial x}(L) \cdot \varphi_{1} \mathrm{~d} t \\
& -\int_{0}^{T} \frac{\partial u}{\partial x}(0) \cdot \varphi_{0} \mathrm{~d} t-\int_{0}^{T} \int_{0}^{L} \frac{\partial^{2} u}{\partial x^{2}} \cdot w \mathrm{~d} x \mathrm{~d} t \\
& =(\tau u)(\varphi)-\int_{0}^{T}\left(\frac{\partial^{2} u}{\partial x^{2}}, w\right)_{L^{2}(\Omega)} \mathrm{d} t
\end{aligned}
$$

We also have:

$$
\int_{0}^{T}\left(\frac{\partial u}{\partial t}, \frac{\partial w}{\partial t}\right)_{L^{2}(\Omega)} \mathrm{d} t=\int_{0}^{L} \frac{\partial u}{\partial t}(T) w(T) \mathrm{d} x-\int_{0}^{T}\left(\frac{\partial^{2} u}{\partial t^{2}}, w\right)_{L^{2}(\Omega)} \mathrm{d} t .
$$

With (2.41), using Fubini's theorem and integration by parts we write:

$$
\begin{gathered}
\int_{0}^{T}\left(\frac{\partial^{2} u}{\partial x^{2}}+\frac{\partial^{2} u}{\partial t^{2}}, w\right)_{L^{2}(\Omega)} \mathrm{d} t=\int_{0}^{T}\left(\frac{\partial^{2} \hat{w}}{\partial t^{2}}-\alpha \frac{\partial \hat{w}}{\partial t}-\frac{\partial^{2} \hat{w}}{\partial x^{2}}+\beta \hat{w} \cos u, w\right)_{L^{2}(\Omega)} \mathrm{d} t \\
=\int_{0}^{T}\left(\hat{w}, \frac{\partial^{2} w}{\partial t^{2}}+\alpha \frac{\partial w}{\partial t}-\frac{\partial^{2} w}{\partial x^{2}}+\beta w \cos u\right)_{L^{2}(\Omega)} \mathrm{d} t \\
\quad+\int_{\Omega} \frac{\partial \hat{w}}{\partial t}(T) w(T) \mathrm{d} x-\int_{0}^{T} \frac{\partial \hat{w}}{\partial x}(L) \varphi_{1} \mathrm{~d} t+\int_{0}^{T} \frac{\partial \hat{w}}{\partial x}(0) \varphi_{0} \mathrm{~d} t \\
=\int_{\Omega} \frac{\partial \hat{w}}{\partial t}(T) w(T) \mathrm{d} x-\int_{0}^{T} \frac{\partial \hat{w}}{\partial x}(L) \varphi_{1} \mathrm{~d} t+\int_{0}^{T} \frac{\partial \hat{w}}{\partial x}(0) \varphi_{0} \mathrm{~d} t
\end{gathered}
$$

Returning to (2.43) we find:

$$
\begin{aligned}
\frac{D \mathcal{J}}{D g}(g) \cdot \varphi= & \int_{0}^{T}(\tau u) \varphi \mathrm{d} t+\int_{\Omega} \frac{\partial u}{\partial t}(T) w(T) \mathrm{d} x-\int_{\Omega} \frac{\partial \hat{w}}{\partial t}(T) w(T) \mathrm{d} x \\
& +\int_{0}^{T} \frac{\partial \hat{w}}{\partial x}(L) \varphi_{1} \mathrm{~d} t-\int_{0}^{T} \frac{\partial \hat{w}}{\partial x}(0) \varphi_{0} \mathrm{~d} t+l(g, \varphi)_{\mathbb{H}^{3}(0, T)} \\
= & \langle\tau u+\tau \hat{w}+l \Lambda g, \varphi\rangle_{\left(\mathbb{H}_{\Gamma}^{3}(0, T)\right)^{\prime}, \mathbb{H}_{\Gamma}^{3}(0, T)} .
\end{aligned}
$$

Hence,

$$
(D \mathcal{J} / D g)(g)=(\tau u+\tau \hat{w}+l \Lambda g),
$$

and since, for an optimal pair $(\bar{g}, \bar{u})$, we have $(D \mathcal{J} / D g)(\bar{g})=0,(2.40)$ follows. 


\subsection{A uniqueness result for the optimal control problem}

We know that if $\mathcal{J}$ is strictly convex the solution of the optimal problem is unique, see e.g. [5]. Our aim is now to show that for $l$ sufficiently large, the cost function

$$
\mathcal{J}(g)=\frac{1}{2} \int_{0}^{T}\left|\frac{\partial u_{g}}{\partial t}\right|_{L^{2}(\Omega)}^{2} \mathrm{~d} t+\frac{1}{2} \int_{0}^{T}\left|\frac{\partial u_{g}}{\partial x}\right|_{L^{2}(\Omega)}^{2} \mathrm{~d} t+\frac{l}{2}|g|_{\mathbb{H}^{3}(0, T)}^{2},
$$

is indeed strictly convex.

Theorem 2.4. Let $u_{0} \in D(A), u_{1} \in V$ and let $\mathcal{J}$ be defined on a bounded, convex, closed, non-empty subset $\mathcal{C}$ of $\mathbb{H}_{\Gamma}^{3}(0, T)$. Then there exists $l_{0}=l\left(u_{0}, u_{1}, \mathcal{C}, T\right)$, such that for any $l \geq l_{0}$, $\mathcal{J}$ is a strictly convex, lower semi-continuous function on $\mathcal{C}$.

Proof. We showed that $g \mapsto \mathcal{J}(g)$ is lower semi-continuous when we proved the existence result for the control problem and it remains to prove that $\mathcal{J}$ is strictly convex. To prove this it is sufficient to prove that the function

$$
f(\rho)=\mathcal{J}(g+\rho \varphi)\left(\mathrm{g}, \varphi \text { arbitrarily chosen in } \mathbb{H}_{\Gamma}^{3}(0, T)\right)
$$

is strictly convex with respect to $\rho$ near $\rho=0$, i.e. $f^{\prime \prime}(0)>0$.

We know that $\left(D u_{g} / D g\right)(\varphi):=w(\varphi)$ is the solution of $(2.41)$. We then compute:

$$
f^{\prime}(\rho)=\frac{D \mathcal{J}}{D g}(g+\rho \varphi) \cdot \varphi=\int_{0}^{T}\left(\frac{\partial u}{\partial t}, \frac{\partial w}{\partial t}\right)_{L^{2}(\Omega)} \mathrm{d} t+\int_{0}^{T}\left(\frac{\partial u}{\partial x}, \frac{\partial w}{\partial x}\right)_{L^{2}(\Omega)} \mathrm{d} t+l(g+\rho \varphi, \varphi)_{\mathbb{H}^{3}(0, T)} .
$$

We then consider $\omega=\left(D^{2} u / D g^{2}\right) \cdot \varphi \cdot q$ and $w^{1}=(D u / D g) \cdot q$. One can show, as in Lemma 2.1, that $\omega$ is the solution of:

$$
\begin{aligned}
& \frac{\partial^{2} \omega}{\partial t^{2}}+\alpha \frac{\partial \omega}{\partial t}-\frac{\partial^{2} \omega}{\partial x^{2}}+\beta \omega \cos u_{g}=\beta w w^{1} \sin u_{g} \\
& \omega(0, t)=0, \quad \omega(L, t)=0 \\
& \omega(x, 0)=0, \quad \frac{\partial \omega}{\partial t}(x, 0)=0
\end{aligned}
$$

We take $q=\varphi$, so that $w^{1}=w$. We then write

$$
f^{\prime \prime}(0)=\int_{0}^{T}\left|\frac{\partial w}{\partial t}\right|_{L^{2}(\Omega)}^{2} \mathrm{~d} t+\int_{0}^{T}\left|\frac{\partial w}{\partial x}\right|_{L^{2}(\Omega)}^{2} \mathrm{~d} t+\int_{0}^{T}\left(\frac{\partial u}{\partial x}, \frac{\partial \omega}{\partial x}\right)_{L^{2}(\Omega)} \mathrm{d} t+\int_{0}^{T}\left(\frac{\partial u}{\partial t}, \frac{\partial \omega}{\partial t}\right)_{L^{2}(\Omega)} \mathrm{d} t+l|\varphi|_{\mathbb{H}^{3}(0, T)}^{2}
$$

We know that

$$
\begin{aligned}
\left|\int_{0}^{T}\left(\frac{\partial u}{\partial x}, \frac{\partial \omega}{\partial x}\right)_{L^{2}(\Omega)} \mathrm{d} t\right| & \leq\left|\frac{\partial u}{\partial x}\right|_{L^{2}\left(0, T ; L^{2}(\Omega)\right)}\left|\frac{\partial \omega}{\partial x}\right|_{L^{2}\left(0, T ; L^{2}(\Omega)\right)} \\
\left|\int_{0}^{T}\left(\frac{\partial u}{\partial t}, \frac{\partial \omega}{\partial t}\right)_{L^{2}(\Omega)} \mathrm{d} t\right| & \leq\left|\frac{\partial u}{\partial t}\right|_{L^{2}\left(0, T ; L^{2}(\Omega)\right)}\left|\frac{\partial \omega}{\partial t}\right|_{L^{2}\left(0, T ; L^{2}(\Omega)\right)}
\end{aligned}
$$

and so we obtain

$$
f^{\prime \prime}(0) \geq-\left|\frac{\partial u}{\partial x}\right|_{L^{2}\left(0, T ; L^{2}(\Omega)\right)}\left|\frac{\partial \omega}{\partial x}\right|_{L^{2}\left(0, T ; L^{2}(\Omega)\right)}-\left|\frac{\partial u}{\partial t}\right|_{L^{2}\left(0, T ; L^{2}(\Omega)\right)}\left|\frac{\partial \omega}{\partial t}\right|_{L^{2}\left(0, T ; L^{2}(\Omega)\right)}+l|\varphi|_{\mathbb{H}^{3}(0, T)}^{2} .
$$


We need to estimate $\omega$, so we multiply equation $(2.47)_{1}$ by $\partial \omega / \partial t$ and we obtain:

$$
\frac{1}{2} \frac{\mathrm{d}}{\mathrm{d} t}\left[\left|\frac{\partial \omega}{\partial t}\right|_{L^{2}(\Omega)}^{2}+\left|\frac{\partial \omega}{\partial x}\right|_{L^{2}(\Omega)}^{2}\right]+\alpha\left|\frac{\partial \omega}{\partial t}\right|_{L^{2}(\Omega)}^{2}=\beta \int_{\Omega} w^{2} \frac{\partial \omega}{\partial t} \sin u_{g} \mathrm{~d} x-\beta \int_{\Omega} \frac{\partial \omega}{\partial t} \omega \cos u_{g} \mathrm{~d} x
$$

We can easily estimate the terms from the RHS of (2.49), using Poincaré's inequality:

$$
\begin{aligned}
& \left|\beta \int_{\Omega} w^{2} \frac{\partial \omega}{\partial t} \sin u_{g} \mathrm{~d} x\right| \leq|\beta| \int_{\Omega}|w|^{2}\left|\frac{\partial \omega}{\partial t}\right| \mathrm{d} x \leq c \int_{\Omega}|w|^{4} \mathrm{~d} x+\frac{\alpha}{4} \int_{\Omega}\left|\frac{\partial \omega}{\partial t}\right|^{2} \mathrm{~d} x, \\
& \left|\beta \int_{\Omega} \frac{\partial \omega}{\partial t} \omega \cos u_{g} \mathrm{~d} x\right| \leq|\beta| \int_{\Omega}|\omega|\left|\frac{\partial \omega}{\partial t}\right| \mathrm{d} x \leq \frac{\alpha}{4}\left|\frac{\partial \omega}{\partial t}\right|_{L^{2}(\Omega)}^{2}+c\left|\frac{\partial \omega}{\partial x}\right|_{L^{2}(\Omega)}^{2} .
\end{aligned}
$$

Returning to (2.49) we find

$$
\frac{\mathrm{d}}{\mathrm{d} t}\left[\left|\frac{\partial \omega}{\partial t}\right|_{L^{2}(\Omega)}^{2}+\left|\frac{\partial \omega}{\partial x}\right|_{L^{2}(\Omega)}^{2}\right]+\alpha\left|\frac{\partial \omega}{\partial t}\right|_{L^{2}(\Omega)}^{2} \leq c \int_{\Omega}|w|^{4} \mathrm{~d} x+c\left|\frac{\partial \omega}{\partial x}\right|_{L^{2}(\Omega)}^{2}
$$

We then need to estimate $w$. We use the lifting function and write $w(x, t)=\gamma(x, t)+\phi_{\varphi}(x, t)$ where $\phi_{\varphi}(x, t)=$ $\varphi_{0}(t)+(x / L)\left(\varphi_{1}(t)-\varphi_{0}(t)\right)$. Then $\gamma$ satisfies:

$$
\begin{aligned}
& \frac{\partial^{2} \gamma}{\partial t^{2}}+\alpha \frac{\partial \gamma}{\partial t}-\frac{\partial^{2} \gamma}{\partial x^{2}}+\beta \gamma \cos u_{g}=\widetilde{F}(x, t) \\
& \gamma(0, t)=0, \quad \gamma(L, t)=0 \\
& \gamma(x, 0)=0, \quad \frac{\partial \gamma}{\partial t}(x, 0)=0
\end{aligned}
$$

where $\widetilde{F}(x, t)=-\left[\partial^{2} \phi / \partial t^{2}+\alpha \partial \phi / \partial t+\beta \phi \cos u_{g}\right]$.

We make the same kind of calculations as before, multiply the first equation of (2.51) by $\partial \phi / \partial t$ and integrate over $\Omega$. We use as before the Gronwall lemma and we obtain:

$$
\left|\frac{\partial \omega}{\partial t}(t)\right|_{L^{2}(\Omega)}^{2}+\left|\frac{\partial \omega}{\partial x}(t)\right|_{L^{2}(\Omega)}^{2} \leq c \int_{0}^{T}|w|_{L^{2}(\Omega)}^{2}|w|_{L^{\infty}(\Omega)}^{2} \mathrm{~d} t
$$

for all $t \leq T$.

We notice that

$$
|w|_{L^{\infty}(\Omega)}^{2} \leq c\left(|\gamma|_{H_{1}^{0}(\Omega)}^{2}+\left|\phi_{\varphi}\right|_{L^{\infty}(\Omega)}^{2}\right), \quad|w|_{L^{2}(\Omega)}^{2} \leq c\left(|\gamma|_{L^{2}(\Omega)}^{2}+\left|\phi_{\varphi}\right|_{L^{2}(\Omega)}^{2}\right)
$$

and so we obtain

$$
\int_{0}^{T}|w|_{L^{2}(\Omega)}^{2}|w|_{L^{\infty}(\Omega)}^{2} \mathrm{~d} t \leq c|\varphi|_{\mathbb{H}^{3}(0, T)}^{4}
$$

We return to (2.52) and we see that:

$$
\int_{0}^{T}\left[\left|\frac{\partial \omega}{\partial t}(t)\right|_{L^{2}(\Omega)}^{2}+\left|\frac{\partial \omega}{\partial x}(t)\right|_{L^{2}(\Omega)}^{2}\right] \mathrm{d} t \leq c|\varphi|_{\mathbb{H}^{3}(0, T)}^{4}
$$


We return to $f^{\prime \prime}(0)$ and using $(2.55)$ we obtain:

$$
\begin{aligned}
f^{\prime \prime}(0) \geq-c\left|\frac{\partial u}{\partial t}\right|_{L^{2}\left(0, T ; L^{2}(\Omega)\right)}|\varphi|_{\mathbb{H}^{3}(0, T)}^{2}-c\left|\frac{\partial u}{\partial x}\right|_{L^{2}\left(0, T ; L^{2}(\Omega)\right)}|\varphi|_{\mathbb{H}^{3}(0, T)}^{2} & \\
+l|\varphi|_{\mathbb{H}^{3}(0, T)}^{2} & =\left(l-c\left|\frac{\partial u}{\partial t}\right|_{L^{2}\left(0, T ; L^{2}(\Omega)\right)}-c\left|\frac{\partial u}{\partial x}\right|_{L^{2}\left(0, T ; L^{2}(\Omega)\right)}\right)|\varphi|_{\mathbb{H}^{3}(0, T)}^{2}
\end{aligned}
$$

The next step consists in bounding $|\partial u / \partial t|_{L^{2}\left(0, T ; L^{2}(\Omega)\right)}$ and $|\partial u / \partial x|_{L^{2}\left(0, T ; L^{2}(\Omega)\right)}$. Using the same estimates as for the proof of Theorem 1.2 we obtain

$$
\begin{aligned}
\left|\frac{\partial v}{\partial t}\right|_{L^{2}(\Omega)}^{2}+\left|\frac{\partial v}{\partial x}\right|_{L^{2}(\Omega)}^{2} & \leq c \int_{0}^{T}|F(x, t)|_{L^{2}(\Omega)}^{2} \mathrm{~d} t+c T \\
& \leq c|g|_{\mathbb{H}^{3}(0, T)}^{2}+c T
\end{aligned}
$$

for all $t \leq T$. From the estimates above and from the fact that $\mathcal{J}$ is defined on a bounded set $\mathcal{C}$ we obtain that:

$$
\left|\frac{\partial u}{\partial t}\right|_{L^{2}\left(0, T ; L^{2}(\Omega)\right)} \leq c, \quad\left|\frac{\partial u}{\partial x}\right|_{L^{2}\left(0, T ; L^{2}(\Omega)\right)} \leq c .
$$

Looking to $(2.56)$, we see that for $l$ big enough, $f^{\prime \prime}(0)>0$, which is what we needed.

Theorem 2.5. Assume that $\mathcal{C}$ and $l_{0}$ are as in Theorem 2.4. Then, for $l \geq l_{0}$, the optimal control problem $\mathcal{P}$ has a unique solution.

\section{Robust CONTROL}

In this section the boundary values are decomposed into the disturbance $h=\left(h_{0}, h_{1}\right) \in \mathbb{H}_{\Gamma}^{3}(0, T)$ and the control $g=\left(g_{0}, g_{1}\right) \in \mathbb{H}_{\Gamma}^{3}(0, T)$. The objective in the robust control problem is to find the best control $g$ in the presence of the worse disturbance $h$ which maximally spoils the control objective. The flow $u$ is related to the disturbance $h$ and the control $g$ through the system:

$$
\begin{aligned}
& \frac{\partial^{2} u}{\partial t^{2}}+\alpha \frac{\partial u}{\partial t}-\frac{\partial^{2} u}{\partial x^{2}}+\beta \sin u=0, \text { in } \Omega \times \mathbf{R}_{+}, \\
& u(0, t)=g_{0}(t)+h_{0}(t), \quad u(L, t)=g_{1}(t)+h_{1}(t), \\
& u(x, 0)=u_{0}(x), \quad \frac{\partial u}{\partial t}(x, 0)=u_{1}(x), \quad x \in \Omega .
\end{aligned}
$$

The cost functional (Lagrangian) considered here is given by:

$\mathcal{J}(g, h)=\frac{1}{2} \int_{0}^{T}\left|\frac{\partial u(g, h)}{\partial t}\right|_{L^{2}(\Omega)}^{2} \mathrm{~d} t+\frac{1}{2} \int_{0}^{T}\left|\frac{\partial u(g, h)}{\partial x}\right|_{L^{2}(\Omega)}^{2} \mathrm{~d} t+\frac{l}{2}|g|_{\mathbb{H}^{3}(0, T)}^{2}-\frac{m}{2}|h|_{\mathbb{H}^{3}(0, T)}^{2}$,

where the scalar parameters $l, m>0$ are given. The parameter $l$ may be interpreted as a measure of the "price" of the control and $m$ as a measure of the "price" of the disturbance.

Definition 3.1. If $(\bar{g}, \bar{h})$ is a saddle point of the cost functional $\mathcal{J}$ defined above, then the disturbance $\bar{h} \in$ $\mathbb{H}_{\Gamma}^{3}(0, T)$, the control $\bar{g} \in \mathbb{H}_{\Gamma}^{3}(0, T)$ and the corresponding solution $\bar{u}(\bar{g}, \bar{h})$ of $(3.1)$ associated to $(\bar{g}, \bar{h})$ are said to solve the robust control problem.

We can solve the robust control problem by using, for instance, the following general result (see e.g., [5]): 
Theorem 3.1. Let $\mathcal{J}$ be a functional defined on $\mathcal{X} \times \mathcal{Y}$, where $\mathcal{X} \subset \mathrm{X}$ and $\mathcal{Y} \subset \mathrm{Y}$ are nonempty, closed, bounded, convex subsets, and $\mathrm{X}, \mathrm{Y}$ are reflexive Banach spaces. If $\mathcal{J}$ satisfies

(a) $\forall g \in \mathcal{X}, h \mapsto \mathcal{J}(g, h)$ is concave and upper semi-continuous,

(b) $\forall h \in \mathcal{Y}, g \mapsto \mathcal{J}(g, h)$ is convex and lower semi-continuous,

then the functional $\mathcal{J}$ has at least one saddle point $(\bar{g}, \bar{h})$ on $\mathcal{X} \times \mathcal{Y}$, such that

$$
\mathcal{J}(\bar{g}, \bar{h})=\min _{g \in \mathcal{X}} \max _{h \in \mathcal{Y}} \mathcal{J}(g, h)=\max _{h \in \mathcal{Y}} \min _{g \in \mathcal{X}} \mathcal{J}(g, h)
$$

Moreover, if $\mathcal{J}$ is strictly convex with respect to $g$ for each $h$ and strictly concave with respect to $h$ for each $g$, then $(\bar{g}, \bar{h})$ is unique.

Proposition 3.1. We assume, in addition to the hypotheses (a) and (b), that

$$
\begin{gathered}
\left(a^{\prime}\right) \forall g \in \mathcal{X}, h \mapsto \mathcal{J}(g, h) \text { is Gâteaux-differentiable, } \\
\left(b^{\prime}\right) \forall h \in \mathcal{Y}, g \mapsto \mathcal{J}(g, h) \text { is Gâteaux-differentiable. }
\end{gathered}
$$

Then $(\bar{g}, \bar{h}) \in \mathcal{X} \times \mathcal{Y}$ is a saddle point of $\mathcal{J}$ if and only if

$$
\begin{aligned}
& \left(\frac{D \mathcal{J}}{D g}(\bar{g}, \bar{h}), g-\bar{g}\right) \geq 0, \forall g \in \mathcal{X}, \\
& \left(\frac{D \mathcal{J}}{D h}(\bar{g}, \bar{h}), h-\bar{h}\right) \leq 0, \forall h \in \mathcal{Y} .
\end{aligned}
$$

If there is no constraint, i.e. $\mathcal{X}=\mathrm{X}, \mathcal{Y}=\mathrm{Y}$, the above inequalities become equalities.

We now return to the robust control problem and we prove that we can apply Theorem 3.1.

Theorem 3.2. Let there be given $u_{0} \in D(A), u_{1} \in V$ and assume that the cost functional $\mathcal{J}$ is defined on $\mathcal{X} \times \mathcal{Y}$, where $\mathcal{X}, \mathcal{Y} \subset \mathbb{H}_{\Gamma}^{3}(0, T)$ are non-empty, closed, convex, bounded sets. Then there exists $l_{0}$ and $m_{0}$ depending on the initial data and on the sets $\mathcal{X}, \mathcal{Y}$ such that, for any $l \geq l_{0}$ and $m \geq m_{0}$ we have:

(a) $\forall g \in \mathcal{X}, h \mapsto \mathcal{J}(g, h)$ is strictly concave and upper semi-continuous,

(b) $\forall h \in \mathcal{Y}, g \mapsto \mathcal{J}(g, h)$ is strictly convex and lower semi-continuous.

Proof. Since the norm is continuous, in order to prove the continuity we only need to verify the continuity of the first two terms in $\mathcal{J}$ with respect to $(g, h)$. Let $u=u(g, h), u_{\star}=u\left(g_{\star}, h_{\star}\right)$ be the solutions of $(3.1)$ associated with the corresponding boundary conditions.

Let $\delta g=g-g_{\star}, \delta h=h-h_{\star}, \delta u=u-u_{\star}$. The lifting function is:

$$
\phi(g, h)(x, t)=g_{0}(t)+h_{0}(t)+\frac{x}{L}\left(g_{1}(t)+h_{1}(t)-g_{0}(t)-h_{0}(t)\right) .
$$

We know that $u(g, h)=v(g, h)+\phi(g, h)$. We denote

$$
\begin{aligned}
\delta v=v(g, h)-v\left(g_{\star}, h_{\star}\right) & =u(g, h)-u\left(g_{\star}, h_{\star}\right)-\phi(g, h)+\phi\left(g_{\star}, h_{\star}\right) \\
& =\delta u-\phi(\delta g, \delta h) .
\end{aligned}
$$


We notice that $\delta v$ is solution of the following system:

$$
\begin{aligned}
& \frac{\partial^{2}(\delta v)}{\partial t^{2}}+\alpha \frac{\partial(\delta v)}{\partial t}-\frac{\partial^{2}(\delta v)}{\partial x^{2}}+\beta \sin (v+\phi(g, h))-\beta \sin \left(v_{\star}+\phi\left(g_{\star}+h_{\star}\right)\right)=\widetilde{F}, \\
& (\delta v)(0, t)=0, \quad(\delta v)(L, t)=0,(\delta v)(x, 0)=0, \quad \frac{\partial(\delta v)}{\partial t}(x, 0)=0,
\end{aligned}
$$

where $\widetilde{F}(x, t)=-\left[\left(\partial^{2} \phi(\delta g, \delta h) / \partial t^{2}\right)(x, t)+\alpha(\partial \phi(\delta g, \delta h) / \partial t)(x, t)\right]$.

We perform the same kind of estimates as in the previous sections and we obtain:

$$
\left|\frac{\partial(\delta v)}{\partial t}(t)\right|_{L^{2}(\Omega)}^{2}+\left|\frac{\partial(\delta v)}{\partial x}(t)\right|_{L^{2}(\Omega)}^{2} \leq c \int_{0}^{T}\left[|\widetilde{F}|_{L^{2}(\Omega)}^{2}+|\phi(\delta g, \delta h)|_{L^{2}(\Omega)}^{2}\right] \mathrm{d} s .
$$

Taking into account the definition of $\widetilde{F}$ and $\phi(\delta g, \delta h)$ we easily find:

$$
\int_{0}^{T}\left[\left|\frac{\partial(\delta v)}{\partial t}(t)\right|_{L^{2}(\Omega)}^{2}+\left|\frac{\partial(\delta v)}{\partial x}(t)\right|_{L^{2}(\Omega)}^{2}\right] \mathrm{d} t \leq c\left[|\delta g|_{\mathbb{H}^{3}(0, T)}^{2}+|\delta h|_{\mathbb{H}^{3}(0, T)}^{2}\right] .
$$

From (3.6) we obtain that

$$
\begin{aligned}
& \forall g \in \mathcal{X}, h \mapsto \mathcal{J}(g, h) \text { is upper semi-continuous, } \\
& \forall h \in \mathcal{Y}, g \mapsto \mathcal{J}(g, h) \text { is lower semi-continuous. }
\end{aligned}
$$

It now remains to prove that

$$
\begin{aligned}
& \forall g \in \mathcal{X}, h \mapsto \mathcal{J}(g, h) \text { is strictly concave, } \\
& \forall h \in \mathcal{Y}, g \mapsto \mathcal{J}(g, h) \text { is strictly convex. }
\end{aligned}
$$

Because the proofs are similar, we only prove that $h \mapsto \mathcal{J}(g, h)$ is strictly concave $\forall g \in \mathcal{X}$.

We introduce the function $f(\rho)=\mathcal{J}\left(g, h+\rho h_{\star}\right)$, where $g, h, h_{\star} \in \mathcal{F}$ are arbitrarily chosen. In order to prove the concavity, it is sufficient to show that $f$ is concave with respect to $\rho$ near $\rho=0$, i.e. $f^{\prime \prime}(0)<0$. Let $w_{\star}\left(0, h_{\star}\right)=\frac{D u}{D h} \cdot h_{\star}$, which is solution of the system:

$$
\left\{\begin{array}{l}
\frac{\partial^{2} w_{\star}}{\partial t^{2}}+\alpha \frac{\partial w_{\star}}{\partial t}-\frac{\partial^{2} w_{\star}}{\partial x^{2}}+\beta w_{\star} \cos u_{g, h}=0, \\
w_{\star}(0, t)=h_{\star}(t), \quad w_{\star}(L, t)=h_{\star}(t), \\
w_{\star}(x, 0)=0, \quad \frac{\partial w_{\star}}{\partial t}(x, 0)=0 .
\end{array}\right.
$$

We then compute:

$f^{\prime}(\rho)=\frac{D \mathcal{J}}{D h}\left(g, h+\rho h_{\star}\right) \cdot h_{\star}=\int_{0}^{T}\left(\frac{\partial u}{\partial t}, \frac{\partial w_{\star}}{\partial t}\right)_{L^{2}(\Omega)} \mathrm{d} t+\int_{0}^{T}\left(\frac{\partial u}{\partial x}, \frac{\partial w_{\star}}{\partial x}\right)_{L^{2}(\Omega)} \mathrm{d} t-m\left(h+\rho h_{\star}, h_{\star}\right)_{\mathbb{H}^{3}(0, T)}$.

We also consider $\widetilde{w}\left(0, h_{\star \star}\right)=\left(D^{2} u / D h^{2}\right) \cdot h_{\star} \cdot h_{\star \star}$, which is solution of the system:

$$
\begin{aligned}
& \frac{\partial^{2} \widetilde{w}}{\partial t^{2}}+\alpha \frac{\partial \widetilde{w}}{\partial t}-\frac{\partial^{2} \widetilde{w}}{\partial x^{2}}+\beta \widetilde{w} \cos u(g, h)=\beta w_{\star} w_{\star \star} \sin u(g, h), \\
& \widetilde{w}(0, t)=0, \quad \widetilde{w}(L, t)=0, \\
& \widetilde{w}(x, 0)=0, \quad \frac{\partial \widetilde{w}}{\partial t}(x, 0)=0,
\end{aligned}
$$


where $w_{\star \star}=(D u / D h) \cdot h_{\star \star}$. Taking $h_{\star}=h_{\star \star}$ we obtain $w_{\star}=w_{\star \star}$ and we can see that $\widetilde{w}\left(0, h_{\star}\right)=\left(D^{2} u / D h^{2}\right)$. $h_{\star} \cdot h_{\star}$ is solution of the system:

$$
\begin{aligned}
& \frac{\partial^{2} \widetilde{w}}{\partial t^{2}}+\alpha \frac{\partial \widetilde{w}}{\partial t}-\frac{\partial^{2} \widetilde{w}}{\partial x^{2}}+\beta \widetilde{w} \cos u(g, h)=\beta w_{\star}^{2} \sin u(g, h), \\
& \widetilde{w}(0, t)=0, \quad \widetilde{w}(L, t)=0, \\
& \widetilde{w}(x, 0)=0, \quad \frac{\partial \widetilde{w}}{\partial t}(x, 0)=0 .
\end{aligned}
$$

We can now compute:

$$
\begin{aligned}
f^{\prime \prime}(0)=\int_{0}^{T}\left|\frac{\partial w_{\star}}{\partial t}\right|_{L^{2}(\Omega)}^{2} \mathrm{~d} t+\int_{0}^{T}\left(\frac{\partial u}{\partial t}, \frac{\partial \widetilde{w}}{\partial t}\right)_{L^{2}(\Omega)} \mathrm{d} t & +\int_{0}^{T}\left|\frac{\partial w_{\star}}{\partial x}\right|_{L^{2}(\Omega)}^{2} \mathrm{~d} t \\
& +\int_{0}^{T}\left(\frac{\partial u}{\partial x}, \frac{\partial \widetilde{w}}{\partial x}\right)_{L^{2}(\Omega)} \mathrm{d} t-m\left|h_{\star}\right|_{\mathbb{H}^{3}(0, T)}^{2} .
\end{aligned}
$$

We show that for $m$ large enough, the last term dominates the expression $(3.10)$ and we obtain $f^{\prime \prime}(0)<0$. To estimate the first terms of $f^{\prime \prime}(0)$ we need to estimate $w_{\star}$ and $\widetilde{w}$.

We first estimate $w_{\star}$. We write $w_{\star}(x, t)=\gamma(x, t)+\phi(x, t)$ where $\phi(x, t)=h_{\star 0}(t)+(x / L)\left(h_{\star 1}(t)-h_{\star 0}(t)\right)$. Then $\gamma$ is solution of the system:

$$
\begin{aligned}
& \frac{\partial^{2} \gamma}{\partial t^{2}}+\alpha \frac{\partial \gamma}{\partial t}-\frac{\partial^{2} \gamma}{\partial x^{2}}+\beta \gamma \cos u(g, h)=\widetilde{F} \\
& \gamma(0, t)=0, \quad \gamma(L, t)=0 \\
& \gamma(x, 0)=0, \quad \frac{\partial \gamma}{\partial t}(x, 0)=0
\end{aligned}
$$

where $\widetilde{F}(x, t)=-\left[\partial^{2} \phi / \partial t^{2}+\alpha \partial \phi / \partial t+\beta \phi \cos u(g, h)\right]$.

By the usual methods we find:

$$
\left|\frac{\partial \gamma}{\partial t}(t)\right|_{L^{2}(\Omega)}^{2}+\left|\frac{\partial \gamma}{\partial x}(t)\right|_{L^{2}(\Omega)}^{2} \leq c \int_{0}^{T}|\widetilde{F}(x, t)|_{L^{2}(\Omega)}^{2} \mathrm{~d} t \leq c\left|h_{\star}\right|_{\mathbb{H}^{3}(0, T)}^{2},
$$

for all $t \leq T$. Remembering that $w_{\star}=\gamma+\phi$, we easily infer from (3.12) that

$$
\int_{0}^{T}\left|\frac{\partial w_{\star}}{\partial t}(t)\right|_{L^{2}(\Omega)}^{2} \mathrm{~d} t+\int_{0}^{T}\left|\frac{\partial w_{\star}}{\partial x}(t)\right|_{L^{2}(\Omega)}^{2} \mathrm{~d} t \leq c\left|h_{\star}\right|_{\mathbb{H}^{3}(0, T)}^{2} .
$$

Next step is to estimate

$$
\left|\int_{0}^{T}\left(\frac{\partial u}{\partial t}, \frac{\partial \widetilde{w}}{\partial t}\right)_{L^{2}(\Omega)} \mathrm{d} t\right| \text { and }\left|\int_{0}^{T}\left(\frac{\partial u}{\partial x}, \frac{\partial \widetilde{w}}{\partial x}\right)_{L^{2}(\Omega)} \mathrm{d} t\right| .
$$

By Schwarz's inequality, it suffices to estimate $\partial u / \partial t, \partial \widetilde{w} / \partial t, \partial u / \partial x, \partial \widetilde{w} / \partial x$ in $L^{2}\left(0, T ; L^{2}(\Omega)\right)$.

For $\widetilde{w}$, we multiply the first equation of (3.9) by $\partial \widetilde{w} / \partial t$ and integrate over $\Omega$. We obtain after some elementary computations:

$$
\frac{\mathrm{d}}{\mathrm{d} t}\left[\left|\frac{\partial \widetilde{w}}{\partial t}\right|_{L^{2}(\Omega)}^{2}+\left|\frac{\partial \widetilde{w}}{\partial x}\right|_{L^{2}(\Omega)}^{2}\right]+\alpha\left|\frac{\partial \widetilde{w}}{\partial t}\right|_{L^{2}(\Omega)}^{2} \leq c\left|\frac{\partial \widetilde{w}}{\partial x}\right|_{L^{2}(\Omega)}^{2}+c \int_{\Omega} w^{4} \mathrm{~d} x .
$$


Noticing that $w_{\star}=\gamma+\phi_{h_{\star}}$, where $\gamma \in H_{0}^{1}(\Omega) \subset L^{\infty}(\Omega)$ and $\phi_{h_{\star}} \in L^{\infty}(\Omega)$, we find

$$
\int_{0}^{T} \int_{\Omega} w_{\star}^{4} \mathrm{~d} x \mathrm{~d} t \leq \int_{0}^{T}\left|w_{\star}\right|_{L^{\infty}(\Omega)}^{2}\left|w_{\star}\right|_{L^{2}(\Omega)}^{2} \mathrm{~d} t \leq c\left|h_{\star}\right|_{\mathbb{H}^{3}(0, T)}^{4} .
$$

Using the Gronwall lemma we obtain:

$$
\int_{0}^{T}\left[\left|\frac{\partial \widetilde{w}}{\partial t}(t)\right|_{L^{2}(\Omega)}^{2}+\left|\frac{\partial \widetilde{w}}{\partial x}(t)\right|_{L^{2}(\Omega)}^{2}\right] \mathrm{d} t \leq c\left|h_{\star}\right|_{\mathbb{H}^{3}(0, T)}^{4}
$$

We now return to $f^{\prime \prime}(0)$ and we find:

$$
f^{\prime \prime}(0) \leq\left|h_{\star}\right|_{\mathbb{H}^{3}(0, T)}^{2}\left(c+c\left|\frac{\partial u}{\partial x}\right|_{L^{2}\left(0, T ; L^{2}(\Omega)\right)}+c\left|\frac{\partial u}{\partial t}\right|_{L^{2}\left(0, T ; L^{2}(\Omega)\right)}-m\right) .
$$

For the estimates of $\partial u / \partial t$ and $\partial u / \partial x$ we can repeat the calculations made in Section 2.3 and we obtain the following estimates:

$$
\begin{aligned}
\left|\frac{\partial u}{\partial t}\right|_{L^{2}(\Omega)}^{2} & \leq c|g|_{\mathbb{H}^{3}(0, T)}^{2}+c|h|_{\mathbb{H}^{3}(0, T)}^{2}+c T, \\
\left|\frac{\partial u}{\partial x}\right|_{L^{2}(\Omega)}^{2} & \leq c|g|_{\mathbb{H}^{3}(0, T)}^{2}+c|h|_{\mathbb{H}^{3}(0, T)}^{2}+c T .
\end{aligned}
$$

We assumed that $\mathcal{J}$ is defined on $\mathcal{X} \times \mathcal{Y}$ where $\mathcal{X}$ and $\mathcal{Y}$ are both bounded. We obtain immediately:

$$
\left|\frac{\partial u}{\partial t}\right|_{L^{2}\left(0, T ; L^{2}(\Omega)\right)} \leq c(T, \mathcal{X}, \mathcal{Y}), \quad\left|\frac{\partial u}{\partial x}\right|_{L^{2}\left(0, T ; L^{2}(\Omega)\right)} \leq c(T, \mathcal{X}, \mathcal{Y}) .
$$

Returning to (3.17) we see that there exists an $m_{0}$ such that, for $m \geq m_{0}, f^{\prime \prime}(0)<0$. Hence $h \mapsto \mathcal{J}(g, h)$ is strictly concave for any $g \in \mathcal{X}$.

We conclude by stating the main result of this section:

Theorem 3.3 (existence and uniqueness of the solution to the robust control problem). Assume that $\mathcal{X}$ and $\mathcal{Y}$ are non-empty, closed, convex, bounded sets; $\mathcal{X}, \mathcal{Y} \subset \mathbb{H}_{\Gamma}^{3}(0, T)$ and that $l \geq l_{0}, m \geq m_{0}$, where $m_{0}$, $l_{0}$ are like in the previous lemma. Then there exists a unique saddle point $(\bar{g}, \bar{h}) \in \mathcal{X} \times \mathcal{Y}$ and the corresponding $\bar{u}=u(\bar{g}, \bar{h})$ such that

$$
\mathcal{J}(\bar{g}, \bar{h})=\min _{g \in \mathcal{X}} \max _{h \in \mathcal{Y}} \mathcal{J}(g, h) .
$$

\section{Miscellaneous Remarks}

We conclude with a remark in the following subsection and an auxiliary result in the next one.

\subsection{Remark concerning the weak solutions of the sine-Gordon equation}

Much of what was done in the previous sections, is valid for weaker solutions of system (1.7), that is $u \in$ $L^{2}([0, T], V)$ with $u^{\prime} \in L^{2}([0, T], H)$. We assume $u_{0} \in V, u_{1} \in H$ and that the boundary conditions are given functions from $\mathbb{H}_{\Gamma}^{2}(0, T)=\left\{v \in \mathbb{H}^{2}(0, T), v(0)=0\right\}$ instead of functions from $\mathbb{H}_{\Gamma}^{3}(0, T)$. All the estimates made for strong solutions are still true because of a technical result from [15]: 
Lemma 4.1. Assume that $w \in L^{2}(0, T ; V), w^{\prime} \in L^{2}(0, T ; H)$ and $w^{\prime \prime}+A w \in L^{2}(0, T ; H)$. Then, after modification on a set of measure zero, $w$ is continuous from $[0, T]$ into $V, w^{\prime}$ is continuous from $[0, T]$ into $H$ and in the sense of distributions on $(0, T)$

$$
2\left(w^{\prime \prime}+A w, w^{\prime}\right)=\frac{\mathrm{d}}{\mathrm{d} t}\left\{\left|w^{\prime}\right|^{2}+a(w, w)\right\} .
$$

However, when working with weak solutions, we are not able to obtain a necessary condition for optimality nor the characterization of the gradient as obtained in the next subsection; hence our choice to work with strong solutions.

\subsection{Identification of the gradients and characterization of the solutions of the robust control problem}

In this section we characterize the gradients of the cost functional with respect to the control $g$ and the disturbance $h$. In Section 3 we saw that the existence of a saddle point $(\bar{g}, \bar{h})$ of the functional $\mathcal{J}$ implies

$$
\begin{aligned}
& \left(\frac{D \mathcal{J}}{D g}(\bar{g}, \bar{h}), g-\bar{g}\right) \geq 0, \forall g \in \mathcal{X}, \\
& \left(\frac{D \mathcal{J}}{D h}(\bar{g}, \bar{h}), h-\bar{h}\right) \leq 0, \forall h \in \mathcal{Y} .
\end{aligned}
$$

We should notice that for a solution $(\bar{g}, \bar{h})$ to the robust control problem we may not have $(D \mathcal{J} / D g)(\bar{g}, \bar{h})=$ $(D \mathcal{J} / D h)(\bar{g}, \bar{h})=0$, as they may be located on the boundary of the domain $\mathcal{X} \times \mathcal{Y}$. We obtain this relation if $(\bar{g}, \bar{h})$ is in the interior of $\mathcal{X} \times \mathcal{Y}$ or if $\mathcal{X}$ and $\mathcal{Y}$ are all of $\mathbb{H}_{\Gamma}^{3}(0, T)$ but for this second variant we do not have the existence of a solution of the robust control problem, as it is essential that $\mathcal{X}$ and $\mathcal{Y}$ are bounded sets.

Differentiation of $\mathcal{J}$ leads to the following expressions:

$$
\begin{aligned}
& \frac{D \mathcal{J}}{D g}(g, h) \cdot \widetilde{g}=\int_{0}^{T}\left(\frac{\partial u}{\partial t}, \frac{\partial w_{\star}}{\partial t}\right)_{L^{2}(\Omega)} \mathrm{d} t+\int_{0}^{T}\left(\frac{\partial u}{\partial x}, \frac{\partial w_{\star}}{\partial x}\right)_{L^{2}(\Omega)} \mathrm{d} t+l(g, \widetilde{g})_{\mathbb{H}^{3}(0, T)}, \\
& \frac{D \mathcal{J}}{D h}(g, h) \cdot \widetilde{h}=\int_{0}^{T}\left(\frac{\partial u}{\partial t}, \frac{\partial \widetilde{w}}{\partial t}\right)_{L^{2}(\Omega)} \mathrm{d} t+\int_{0}^{T}\left(\frac{\partial u}{\partial x}, \frac{\partial \widetilde{w}}{\partial x}\right)_{L^{2}(\Omega)} \mathrm{d} t-m(h, \widetilde{h})_{\mathbb{H}^{3}(0, T)},
\end{aligned}
$$

where $w_{\star}=(D u / D g) \cdot \widetilde{g}$ and $\widetilde{w}=(D u / D h) \cdot \widetilde{h}$.

We also introduce the following adjoint state equation:

$$
\begin{aligned}
& \frac{\partial^{2} \hat{w}}{\partial t^{2}}-\alpha \frac{\partial \hat{w}}{\partial t}-\frac{\partial^{2} \hat{w}}{\partial x^{2}}+\beta \hat{w} \cos u=\frac{\partial^{2} u}{\partial t^{2}}+\frac{\partial^{2} u}{\partial x^{2}} \\
& \hat{w}(0, t)=0, \quad \hat{w}(L, t)=0 \\
& \hat{w}(x, T)=0, \quad \frac{\partial \hat{w}}{\partial t}(x, T)=\frac{\partial u}{\partial t}(x, T) .
\end{aligned}
$$

Proposition 4.1. Let $u(g, h)$ be the solution of system (3.1) and let $w=(D u / D g) \cdot \widetilde{g}+(D u / D h) \cdot \widetilde{h}$ be the solution of the following system:

$$
\begin{aligned}
& \frac{\partial^{2} w}{\partial t^{2}}+\alpha \frac{\partial w}{\partial t}-\frac{\partial^{2} w}{\partial x^{2}}+\beta w \cos u=0, \\
& w(0, t)=\widetilde{g}_{0}+\widetilde{h}_{0}, \quad w(L, t)=\widetilde{g}_{1}+\widetilde{h}_{1}, \\
& w(x, 0)=0, \quad \frac{\partial w}{\partial t}(x, 0)=0 .
\end{aligned}
$$


Let $\hat{w}$ be the solution of the adjoint state equation (4.4). Then we obtain:

$$
\int_{0}^{T}\left(\frac{\partial^{2} u}{\partial t^{2}}+\frac{\partial^{2} u}{\partial x^{2}}, w\right)_{L^{2}(\Omega)} \mathrm{d} t=\int_{\Omega} \frac{\partial u}{\partial t}(T) w(T) \mathrm{d} x-\int_{0}^{T} \frac{\partial \hat{w}}{\partial x}(L)\left(\widetilde{g}_{1}+\widetilde{h}_{1}\right) \mathrm{d} t+\int_{0}^{T} \frac{\partial \hat{w}}{\partial x}(0)\left(\widetilde{g}_{0}+\widetilde{h}_{0}\right) \mathrm{d} t
$$

Proof. The proof follows immediately using elementary computations based on integrations by parts.

As in Section 2.3, we introduce the operator $\tau$ defined on $H^{2}(\Omega), \tau u=\left(-\frac{\partial u}{\partial x}(0), \frac{\partial u}{\partial x}(L)\right)$. With this notation we can rewrite $(4.6)$ as

$$
\int_{0}^{T}\left(\frac{\partial^{2} u}{\partial t^{2}}+\frac{\partial^{2} u}{\partial x^{2}}, w\right)_{L^{2}(\Omega)} \mathrm{d} t=\int_{\Omega} \frac{\partial u}{\partial t}(T) w(T) \mathrm{d} x-\int_{0}^{T} \tau(\hat{w}) \cdot(\widetilde{g}+\widetilde{h}) \mathrm{d} t .
$$

Returning to $(4.2)$ and noticing that $w_{\star}=w(\widetilde{g}, 0)$ we obtain:

$$
\begin{aligned}
\frac{D \mathcal{J}}{D g}(g, h) \cdot \widetilde{g}= & \int_{0}^{T}\left(\frac{\partial u}{\partial t}, \frac{\partial w_{\star}}{\partial t}\right)_{L^{2}(\Omega)} \mathrm{d} t+\int_{0}^{T}\left(\frac{\partial u}{\partial x}, \frac{\partial w_{\star}}{\partial x}\right)_{L^{2}(\Omega)} \mathrm{d} t+l(g, \widetilde{g})_{\mathbb{H}^{3}(0, T)} \\
= & \int_{\Omega} \frac{\partial u}{\partial t}(T) w_{\star}(T) \mathrm{d} x+\int_{0}^{T} \frac{\partial u}{\partial x}(L) \widetilde{g}_{1} \mathrm{~d} t-\int_{0}^{T} \frac{\partial u}{\partial x}(0) \widetilde{g}_{0} \mathrm{~d} t \\
& -\int_{0}^{T}\left(\frac{\partial^{2} u}{\partial t^{2}}+\frac{\partial^{2} u}{\partial x^{2}}, w_{\star}\right)_{L^{2}(\Omega)} \mathrm{d} t+l(g, \widetilde{g})_{\mathbb{H}^{3}(0, T)} .
\end{aligned}
$$

Taking $\widetilde{h}=0$ in (4.7) we obtain:

$$
\frac{D \mathcal{J}}{D g}(g, h) \cdot \widetilde{g}=\int_{0}^{T}(\tau u) \widetilde{g} \mathrm{~d} t+\int_{0}^{T}(\tau \hat{w}) \widetilde{g} \mathrm{~d} t+l(g, \widetilde{g})_{\mathbb{H}^{3}(0, T)}
$$

Taking an arbitrary $\widetilde{g}$ we find the expression for the gradient $D \mathcal{J} / D g$ :

$$
\frac{D \mathcal{J}}{D g}(g, h)=\tau u+\tau \hat{w}+l \Lambda g
$$

Similarly we find:

$$
\frac{D \mathcal{J}}{D h}(g, h)=\tau u+\tau \hat{w}-m \Lambda h .
$$

We can now state the main result of this section:

Theorem 4.1. For $m$ and $l$ large enough, the solution to the robust control exists and is unique. Furthermore, the gradients of the cost functional are given by $(D \mathcal{J} / D g)(g, h)=\tau u+\tau \hat{w}+l \Lambda g$ and $(D \mathcal{J} / D h)(g, h)=$ $\tau u+\tau \hat{w}-m \Lambda h$, where $(u, \hat{w})$ is the solution of the following system:

$$
\begin{aligned}
& \frac{\partial^{2} u}{\partial t^{2}}+\alpha \frac{\partial u}{\partial t}-\frac{\partial^{2} u}{\partial x^{2}}+\beta \sin u=0, \\
& \frac{\partial^{2} \hat{w}}{\partial t^{2}}-\alpha \frac{\partial \hat{w}}{\partial t}-\frac{\partial^{2} \hat{w}}{\partial x^{2}}+\beta \hat{w} \cos u=\frac{\partial^{2} u}{\partial t^{2}}+\frac{\partial^{2} u}{\partial x^{2}}, \\
& u(0, t)=g_{0}+h_{0}, \quad u(L, t)=g_{1}+h_{1}, \quad \hat{w}(0, t)=0, \quad \hat{w}(L, t)=0, \\
& u(x, 0)=u_{0}, \quad \frac{\partial u}{\partial t}(x, 0)=u_{1}, \quad \hat{w}(x, T)=0, \quad \frac{\partial \hat{w}}{\partial t}(x, T)=\frac{\partial u}{\partial t}(x, T) .
\end{aligned}
$$


Proof. The existence and uniqueness of the solution to the robust control problem are given in Section 3 . The other statements have been already proven in this section.

Acknowledgements. This work was supported in part by the National Science Foundation under grant NSF-DMS-0074334, NSF-DMS-0305110 and by the Research Fund of Indiana University. The authors thank the anonymous referee for the remarks made.

\section{REFERENCES}

[1] F. Abergel and R. Temam, On some control problems in fluid mechanics. Theor. Comput. Fluid Dyn. 1 (1990) 303-325.

[2] G.P. Agrawal, Nonlinear Fiber Optics. 2nd ed., Academic, San Diego, California (1995).

[3] T.R. Bewley, R. Temam and M. Ziane, A general framework for robust control in fluid mechanics. Physica D 138 (2000) 360-392.

[4] R.W. Boyd, Nonlinear Optics. Academic, Boston (1992).

[5] I. Ekeland and R. Temam, Convex Analysis and Variational Problems. Classics. Appl. Math. 28 (1999).

[6] M. Gunzburger, Adjoint equation-based methods for control problems in incompressible, viscous flows. Flow Turbul. Combust. 65 (2000) 249-272.

[7] M. Gunzburger and O. Yu. Imanuvilov, Optimal control of stationary, Iow Mach number, highly nonisothermal, viscous flows. ESAIM: COCV $\mathbf{5}$ (2000) 477-500.

[8] M. Green and D.J.N. Limebeer, Linear robust control. Pretice-Hall (1995).

[9] C. Hu and R. Temam, Robust control of the Kuramoto-Sivashinsky equation. Dynam. Cont. Discrete Impuls Systems B 8 (2001) 315-338.

[10] J.L. Lions, Problèmes aux limites dans les equations aux dérivées partielles. Presses de l'Université de Montreal (1965), reedited in 2002 as part of [11].

[11] J.L. Lions, Selected work. 3 volumes, EDP Sciences, Paris, France (2003).

[12] M. Marion, Attractors for reaction-diffusion equations; Existence and estimate of their dimension. Appl. Anal. 25 (1987) 101-147.

[13] J. Simon, Compact sets in space $L^{p}(0, T ; B)$. Ann. Mat. Pura Appl. 4 (1987) 67-96.

[14] R. Temam, Navier-Stokes Equations. North-Holland, Amsterdam (1977), reedited in the series: AMS Chelsea, AMS Providence (2001).

[15] R. Temam, Infinite Dimensional Dynamical Systems in Mechanics and Physics. Appl. Math. Sci. 68, Second augmented edition, Springer-Verlag, New York (1997). 